Zhen-Qing Chen · Renming Song

\title{
Conditional gauge theorem for non-local Feynman-Kac transforms
}

Received: 3 July 2001 / Revised version: 10 January 2002 /

Published online: 10 September 2002 - (C) Springer-Verlag 2002

\begin{abstract}
Feynman-Kac transforms driven by discontinuous additive functionals are studied in this paper for a large class of Markov processes. General gauge and conditional gauge theorems are established for such transforms. Furthermore, the $L^{2}$-infinitesimal generator of the Schrödinger semigroup given by a non-local Feynman-Kac transform is determined in terms of its associated bilinear form.
\end{abstract}

\section{Introduction}

Markov processes with discontinuous sample paths constitute an important family of stochastic processes in probability theory. It is well known that (cf. e.g., Janicki and Weron [28], Samorodnitsky and Taqqu [37]) many physical and economic systems should be and in fact have been successfully modeled by discontinuous processes, such as stable processes. In this paper we study FeynmanKac transforms of discontinuous Markov processes through discontinuous additive functionals. In other words, we study non-local perturbations of discontinuous Markov processes and their properties. In this paper we are mainly concerned with the gauge and conditional gauge theorems for this kind of non-local Feynman-Kac transforms. We now explain what the gauge and conditional gauge theorems are while laying out the settings we shall work in.

Let $E$ be a Lusin space (i.e., a space that is homeomorphic to a Borel subset of a compact metric space) and $\mathcal{B}(E)$ be the Borel $\sigma$-algebra on $E$, and let $m$ be a $\sigma$ finite measure on $\mathcal{B}(E)$ with $\operatorname{supp}[m]=E$. Let $X=\left(\Omega, \mathcal{M}, \mathcal{M}_{t}, X_{t}, \mathbf{P}_{x}, x \in\right.$ $E$ ) be a Borel standard process on $E$, which is transient in the sense of [23]. Here, a Borel standard process on Lusin space $E$ is a strong Markov process satisfying the following conditions: (i) it is right continuous, (ii) it has no branching points, (iii) its resolvents map Borel functions into Borel functions and (iv) it is

Z.-Q. Chen: Department of Mathematics, University of Washington, Seattle, WA 98195, USA. e-mail: zchen@math.washington.edu

The research of this author is supported in part by NSF Grant DMS-0071486.

R. Song: Department of Mathematics, University of Illinois, Urbana, IL 61801, USA. e-mail: rsong@math.uiuc.edu

The research of this author is supported in part by NSF Grant DMS-9803240

Mathematics Subject Classification (2000): Primary 60J45, 60J40; Secondary 35J10, 47J20

Key words or phrases: Green function - Conditional Markov process - Kato class - Conditional gauge theorem - Gauge Theorem 
quasi-left continuous on $(0, \zeta)$, where $\zeta$ is the lifetime of the process. The shift operators $\theta_{t}, t \geq 0$, satisfy $X_{s} \circ \theta_{t}=X_{s+t}$ identically for $s, t \geq 0$. Adjoined to the state space $E$ is an isolated point $\partial \notin E$; the process $X$ retires to $\partial$ at its "lifetime" $\zeta:=\inf \left\{t \geq 0: X_{t}=\partial\right\}$. Denote $E \cup\{\partial\}$ by $E_{\partial}$. Throughout this paper, the process $X$ is assumed to be $m$-irreducible in the sense that if a measurable set $A$ has positive $m$-measure then $\mathbf{P}_{x}\left[T_{A}<\infty\right]>0$ for all $x \in E$, where $T_{A}=\inf \left\{t>0, X_{t} \in A\right\}$ is the first hitting time of $A$.

The transition operators $P_{t}, t \geq 0$, are defined by

$$
P_{t} f(x):=\mathbf{E}_{x}\left[f\left(X_{t}\right)\right]=\mathbf{E}_{x}\left[f\left(X_{t}\right) ; t<\zeta\right] .
$$

(Here and in the sequel, unless mentioned otherwise, we use the convention that a function defined on $E$ takes the value 0 at the cemetery point $\partial$.) We assume that there is a Borel function $G(x, y)$ on $E \times E$ such that

$$
\mathbf{E}_{x}\left[\int_{0}^{\infty} f\left(X_{s}\right) d s\right]=\int_{E} G(x, y) f(y) m(d y)
$$

for all measurable $f \geq 0$. $G(x, y)$ is called the Green function of $X$.

For any continuous additive functional of the form

$$
A(t)=\int_{0}^{t} q\left(X_{s}\right) d s,
$$

where $q: E \rightarrow[-\infty, \infty]$ is a Borel function, define the gauge function $g: E \rightarrow$ $[0, \infty]$ by

$$
g(x):=\mathbf{E}_{x}[\exp (A(\zeta))]
$$

The previously known gauge theorem is a result of the following type.

Gauge Theorem. Under suitable hypotheses on the process $X$ and the function $q$, if $g$ is finite at some point $x \in E$, then $g$ is bounded on $E$.

The gauge theorem of this type has been proved for quite general Markov processes in Chung and Rao [14]. See also [43].

Suppose we now have another transient Borel standard process $\widehat{X}=\left(\widehat{\Omega}, \widehat{\mathcal{M}}, \widehat{\mathcal{M}}_{t}\right.$, $\left.\widehat{X}_{t}, \widehat{\mathbf{P}}_{x}, x \in E\right)$ on the same state space $E$ which is a strong dual of $X$ with respect to the measure $m$. That is, the semigroup $\left\{\widehat{P}_{t}\right\}_{t \geq 0}$ of $\widehat{X}$ is the dual in $L^{2}(E, m)$ to the semigroup $\left\{P_{t}\right\}_{t \geq 0}$ of $X$ :

$$
\int_{E} f(x) P_{t} g(x) m(d x)=\int_{E} g(x) \widehat{P}_{t} f(x) m(d x) \text { for all } f, g \in L^{2}(E, m)
$$

and the resolvents $\left\{U_{\alpha}\right\}$ and $\left\{\widehat{U}_{\alpha}\right\}$ satisfy the following conditions: for each $\alpha>0$, a $\mathcal{B}(E \times E)$-measurable potential density $G_{\alpha}(x, y)$ can be chosen so that

(a) $U_{\alpha}(x, d y)=G_{\alpha}(x, y) m(d y), \quad \widehat{U}_{\alpha}(x, d y)=G_{\alpha}(y, x) m(d y)$;

(b) $x \rightarrow G_{\alpha}(x, y)$ is $\alpha$-excessive for $X, \quad y \rightarrow G_{\alpha}(x, y)$ is $\alpha$-excessive for $\widehat{X}$.

When $\alpha=0$, we will drop the subscript and write $G$ for $G_{0}$. 
Under the above assumption, one can easily show that every excessive function of $X$ is Borel measurable. For any excessive function $h$ of $X$, let $E_{h}:=\{x \in E$ : $0<h(x)<\infty\}$ and define

$$
p^{h}(t, x, d y)=\frac{h(y) p(t, x, d y)}{h(x)}, \quad t>0, x, y \in E,
$$

then $p^{h}$ is a transition probability and determines a Borel standard process $X^{h}$ on $E_{h}$ (see [32] or Proposition 2.2 of [16]), which is called Doob's $h$-transformed process of $X$ or the $h$-conditioned process. We are going to use $\zeta^{h}$ to denote the lifetime of the $h$-conditioned process. For any $x \in E$, we are going to use $\mathbf{P}_{x}^{h}$ and $\mathbf{E}_{x}^{h}$ to denote the probability and expectation with respect to the $h$-conditioned process starting from $x$ respectively. When $h(\cdot)=G(\cdot, y)$ for some $y \in E$, we will use $\mathbf{P}_{x}^{y}$ and $\mathbf{E}_{x}^{y}$ to denote the probability and expectation for the $h$-conditioned process starting from $x$ respectively. In this case, the lifetime $\zeta^{h}$ will be denoted as $\zeta^{y}$.

For any continuous additive functional of the form $A(t)=\int_{0}^{t} q\left(X_{s}\right) d s$, where $q: E \rightarrow[-\infty, \infty]$ is an $m$-a.e. finite Borel function, we define the conditional gauge function: $u: E \times E \rightarrow[0, \infty]$ by

$$
u(x, y):=\mathbf{E}_{x}^{y}\left[\exp \left(A\left(\zeta^{y}\right)\right)\right]
$$

All the previously known conditional gauge theorems are of the following type.

Conditional Gauge Theorem. Under suitable hypotheses on $X$ and $q$, if $u$ is finite at some point $(x, y) \in(E \times E) \backslash d$, then $u$ is bounded on $(E \times E) \backslash d$, where $d=\{(x, y) \in E \times E: G(x, y)=0$ or $G(x, y)=\infty\}$.

Unlike the gauge theorem, the conditional gauge theorem had been proved only for a very limited class of symmetric Markov processes, mainly for Brownian motion and symmetric stable processes in bounded Lipschitz domains. The conditional gauge theorem was first proved for Brownian motions by several authors under various conditions (see, Chung and Zhao [15] for a history). The conditional gauge theorem for symmetric stable process was first proved by Chen and Song in [11] on bounded $C^{1,1}$-domains. It was later extended to symmetric stable processes in bounded Lipschitz domains by Bogdan and Byczkowski [7], and by Chen and Song [12] independently, and in more general $\kappa$-fat domains by Song and $\mathrm{Wu}$ [41]. We should point out that in these papers [11]-[12], [7] and [41], the conditional gauge theorem were established in the following slightly weaker form: Under suitable conditions, if the gauge function for the symmetric stable process is finite, then the conditional gauge function is bounded. Very recently general condition gauge theorems were established in Chen and Song [13] for a large class of non-symmetric Markov processes in the ultimate form mentioned above.

When the underlying process $X$ is discontinuous, there is a large class of additive functionals which are discontinuous. In this paper we will be mainly concerned with additive functionals of the following form:

$$
A_{q+F}(t)=\int_{0}^{t} q\left(X_{S}\right) d s+\sum_{s \leq t} F\left(X_{s-}, X_{s}\right),
$$


where $q$ is a Borel function on $E$ and $F$ is some Borel function on $E \times E_{\partial}$ vanishing on the diagonal. We set $e_{q+F}(t)=\exp \left(A_{q+F}(t)\right)$ for convenience. Throughout this paper, all additive functionals should be understood in the strict sense, i.e., in the sense of [6]. The purpose of this paper is to establish gauge and conditional gauge theorems for the gauge function $g(x)=\mathbf{E}_{x}\left[A_{q+F}(\zeta)\right]$ and the conditional gauge function $u(x, y)=\mathbf{E}_{x}^{y}\left[A_{q+F}\left(\zeta^{y}\right)\right]$. As we mentioned above, for gauge and conditional gauge theorems, up to now almost all the attention has been focused on continuous additive functionals of $X$. The only exception is [39], where a gauge theorem was proved for discontinuous additive functionals of a symmetric stable process.

Throughout this paper, we denote by $(N, H)$ a Lévy system of $X$ (cf. [5] and [38]). That is, $H$ is a positive continuous additive functional (PCAF in abbreviation) of $X$ with bounded 1-potential, $N(x, d y)$ is a kernel from $(E, \mathcal{B}(E))$ to $\left(E_{\partial}, \mathcal{B}\left(E_{\partial}\right)\right)$ with $N(x,\{x\})=0$ for all $x \in E$ such that for any nonnegative Borel function $f$ on $E \times E_{\partial}$ vanishing on the diagonal and any $x \in E$,

$$
\mathbf{E}_{x}\left(\sum_{s \leq t} f\left(X_{s-}, X_{s}\right)\right)=\mathbf{E}_{x}\left(\int_{0}^{t} \int_{E_{\partial}} f\left(X_{s}, y\right) N\left(X_{s}, d y\right) d H_{s}\right) .
$$

In this paper, we assume that $H_{s}$ is of the form of $\int_{0}^{s} h\left(X_{s}\right) d s$ for some Borel function $h \geq 0$ on $E$. Many processes such as rotationally symmetric stable processes have such property. We are going to use $\mu_{H}$ to denote the Revuz measure $h(x) m(d x)$ of $H$. When $m$ is an excessive measure of $X, \mu_{H}$ can be characterized as the unique measure (cf. [19]) such that

$$
\int_{E} f(x) \mu_{H}(d x)=\uparrow \lim _{t \downarrow 0} \mathbf{E}_{m}\left[\frac{1}{t} \int_{0}^{t} f\left(X_{s}\right) d H_{s}\right] .
$$

for any Borel measurable function $f \geq 0$. Here $\uparrow \lim _{t \downarrow 0}$ means the quantity is increasing as $t \downarrow 0$.

We remark here that Lévy systems are crucial in dealing with discontinuous additive functionals and our hypothesis that $X$ is a Borel standard process is to guarantee the existence of a Lévy system $(N, H)$. If we are only concerned with continuous additive functionals (i.e. $F=0$ in $A_{q+F}$ ), then we only need to assume that $X$ is a Borel right process having left limits on $(0, \zeta)$ (see [13]).

The content of this paper is organized as follows. In sections 2 and 3, we establish the general gauge and conditional gauge theorems for discontinuous additive functionals, extending the results in [13] where the corresponding results are established for continuous additive functionals. In the last section, the generators of the Schrödinger semigroups obtained from this type of non-local Feynman-Kac transforms are characterized in terms of the associated bilinear form.

\section{Gauge theorem}

In this section, $X$ is an irreducible transient Borel standard process on a Lusin space $E$ with Green function $G(x, y)$, as is specified at the beginning of Section 1. The 
results of this section are generalizations of the corresponding results in [13] to discontinuous additive functionals. They also extend results in [39], where a gauge theorem is proved for $A_{F}$ of a symmetric stable process. Our argument here is adapted from the approach in [13].

The following definition is taken from [13]. For a subset $K \subset E$, we use $K^{c}:=E \backslash K$ to denote the complement of $K$ in $E$.

Definition 2.1. (1) A function $q$ is said to be in the Kato class $\mathbf{K}(X)$ if

$$
\lim _{t \rightarrow 0} \sup _{x \in E} \mathbf{E}_{x}\left[\int_{0}^{t}\left|q\left(X_{s}\right)\right| d s\right]=0 .
$$

(2) A function $q$ is said to be in the class $\mathbf{K}_{\infty}(X)$ if for any $\varepsilon>0$, there is a set $K=K(\varepsilon)$ of finite $m$-measure and a constant $\delta=\delta(\varepsilon)>0$ such that for all measurable set $B \subset E$ with $m(B)<\delta$,

$$
\sup _{x \in E} \int_{K^{c} \cup B} G(x, y)|q(y)| m(d y)<\varepsilon .
$$

(3) A function $q$ is said to be in the class $\mathbf{K}_{1}(X)$ if there is a set $K$ offinite $m$-measure and a constant $\delta>0$ such that

$$
\beta(q):=\sup _{B \subset K: m(B)<\delta}\left(\sup _{x \in E} \int_{K^{c} \cup B} G(x, y)|q(y)| m(d y)\right)<1 .
$$

(4) A measure $v$ given by $v(d x)=q(x) m(d x)$ is said to be in $\mathbf{K}(X), \mathbf{K}_{\infty}(X)$, or $\mathbf{K}_{1}(X)$, if $q$ is in the corresponding classes.

In [4], Amor and Hansen introduced a class of positive measures which they call $G$-Kato measures. For any measure $v=q(x) m(d x)$, define $G v(x):=G q(x)=$ $\int_{E} G(x, y) q(y) m(d y)$. It is easy to see the following.

Proposition 2.1. A positive measure $v$ that is absolutely continuous with respect to the measure $m$ is in $\mathbf{K}_{\infty}(X)$ if and only if it is a $G$-Kato measure, that is, $G v(x)$ is bounded and for every sequence of Borel sets $A_{n}$ that increases to $E, G\left(1_{A_{n}} v\right)$ increases to $G v$ uniformly on $E$.

Proof. Suppose that $v(d x)=q(x) m(d x)$ is a positive measure in $\mathbf{K}_{\infty}(X)$. By Proposition 2.1 of [13], $G v$ is bounded. Let $A_{n}$ be a sequence of Borel sets that increases to $E$. For any given $\varepsilon>0$, let $K=K(\varepsilon)$ and $\delta=\delta(\varepsilon)>0$ be specified as in Definition 2.1 of $\mathbf{K}_{\infty}(X)$ for $v$. As $\lim _{n \rightarrow \infty} m\left(K \backslash A_{n}\right)=0$, there is an integer $N>0$ such that $m\left(K \backslash A_{n}\right)<\delta$ for $n>N$. Hence for $n>N$,

$$
\begin{aligned}
\sup _{x \in E}\left|G v(x)-G\left(1_{A_{n}} v\right)(x)\right| \leq & \sup _{x \in E} \int_{E \backslash K} G(x, y) v(d y) \\
& +\sup _{x \in E} \int_{K \backslash A_{n}} G(x, y) v(d y)<2 \varepsilon .
\end{aligned}
$$

This says that $G\left(1_{A_{n}} v\right)$ increases to $G v$ uniformly on $E$ and so $v$ is $G$-Kato measure in the sense of [4]. 
Conversely, suppose now that $v$ is a $G$-Kato class measure that is absolutely continuous with respect to the measure $m$. Since one can exhaust $E$ by an increasing sequence of Borel sets of finite $m$-measure, for any give $\varepsilon>0$, there is a Borel set $K$ of finite $m$-measure such that

$$
\sup _{x \in E} \int_{E \backslash K} G(x, y) v(d y)<\varepsilon .
$$

We claim that there exists a $\delta>0$ such that for all Borel subset $B \subset K$ with $m(B)<\delta$,

$$
\int_{B} G(x, y) \nu(d y)<\varepsilon .
$$

If this is not true, then one can find a decreasing sequence of Borel subsets $B_{n} \subset K$ with $m\left(B_{n}\right) \leq 2^{-n}$ so that $\sup _{x \in E} \int_{B_{n}} G(x, y) v(d y) \geq \varepsilon$. However $A_{n}:=(E \backslash$ $\left.B_{n}\right) \bigcup\left(\bigcap_{k=1}^{\infty} B_{k}\right)$ is a sequence of Borel sets that increases to $E$. As $v$ is a $G$-Kato measure and is absolutely continuous with respect to $m, G\left(1_{B_{n}} v\right)=G\left(1_{E \backslash A_{n}} v\right)$ should decrease to zero uniformly on $E$, which is a contradiction. Therefore $v$ is in class $\mathbf{K}_{\infty}(X)$.

By Proposition 2.2 of [13], we know $\mathbf{K}_{\infty}(X) \subset \mathbf{K}(X)$. Recall that $\mu_{H}$ is assumed to be absolutely continuous with respect to the reference measure $m$.

Definition 2.2. Suppose that $F$ is a Borel function on $E \times E_{\partial}$ vanishing on the diagonal.

(1) We say that $F$ belongs to the class $\mathbf{J}(X)$ if $F$ is bounded and the measure $N|F|(x) \mu_{H}(d x)$ belongs to $\mathbf{K}(X)$, where

$$
N|F|(x):=\int_{E_{\partial}}|F(x, y)| N(x, d y) .
$$

(2) We say that $F$ belongs to the class $\mathbf{J}_{\infty}(X)$ if $F$ is bounded and the measure $N|F|(x) \mu_{H}(d x)$ belongs to $\mathbf{K}_{\infty}(X)$.

It is easy to see that if $F_{1}$ and $F_{2}$ belong to $\mathbf{J}(X)$ (respectively $\mathbf{J}_{\infty}(X)$ ) and $c$ is a constant, then $c F_{1}, F_{1}+F_{2}, F_{1} F_{2}$ all belong to $\mathbf{J}(X)$ (respectively $\mathbf{J}_{\infty}(X)$ ). Furthermore, one can also show easily that if $F$ belongs to $\mathbf{J}(X)$ (respectively $\mathbf{J}_{\infty}(X)$ ), then the function $e^{F}-1$ belongs to $\mathbf{J}(X)$ (respectively $\mathbf{J}_{\infty}(X)$ ).

Proposition 2.2. If $q \in \mathbf{K}_{1}(X)$ and $F \in \mathbf{J}_{\infty}(X)$, then the function

$$
x \mapsto \mathbf{E}_{x}\left[A_{|q|+|F|}(\zeta)\right], \quad x \in E
$$

is bounded.

Proof. For any $x \in E$,

$\mathbf{E}_{x}\left[A_{|q|+|F|}(\zeta)\right]=\mathbf{E}_{x}\left[\int_{0}^{\zeta}|q|\left(X_{s}\right) d s\right]+\mathbf{E}_{x}\left[\int_{0}^{\zeta} \int_{E_{\partial}}\left|F\left(X_{s}, y\right)\right| N\left(X_{s}, d y\right) d H_{s}\right]$. 
As $q \in \mathbf{K}_{1}(X)$ and $N|F|(x) \mu_{H}(d x)$ belongs to $\mathbf{K}_{\infty}(X)$, our assertion follows from Proposition 2.1 of [13].

This proposition implies that for $q \in \mathbf{K}_{1}(X)$ and $F \in \mathbf{J}_{\infty}(X), A_{q+F}(\zeta)$ is almost surely finite and so $e_{q+F}(\zeta)$ is finite almost surely. Hence the gauge function

$$
g(x)=\mathbf{E}_{x}\left[e_{q+F}(\zeta)\right], \quad x \in E
$$

is well defined, nonnegative. Since $\zeta$ is in the $\sigma$-field $\sigma\left\{X_{t}, t \geq 0\right\}$, one can check easily that $g$ is Borel measurable.

Proposition 2.3. For $q \in \mathbf{K}_{1}(X) \cup \mathbf{K}(X)$ and $F \in \mathbf{J}(X)$, there exist positive constants $c_{1}, \beta_{1}$ such that

$$
\sup _{x \in E} \mathbf{E}_{x}\left[e_{q+F}(t)\right] \leq c_{1} e^{\beta_{1} t} \quad \text { for all } t \geq 0 .
$$

Proof. We need only to prove the assertion for nonnegative $q$ and $F$. For $q \in \mathbf{K}_{1}(X)$, there is a constant $\alpha>1$ such that $\alpha q \in \mathbf{K}_{1}(X)$. Let $\gamma>1$ be such that $\frac{1}{\alpha}+\frac{1}{\gamma}=1$. Put $F_{1}=e^{2 \gamma F}-1$ and

$$
B_{t}=\sum_{s \leq t} F_{1}\left(X_{s-}, X_{s}\right)
$$

Then

$$
B_{t}^{p}:=\int_{0}^{t} \int_{E_{\partial}} F_{1}\left(X_{s}, y\right) N\left(X_{s}, d y\right) d H_{s}
$$

is the dual predictable projection of $B_{t}$. Therefore $B_{t}-B_{t}^{p}$ is a $\mathbf{P}_{x}$-martingale for every $x \in E$. Now it follows from the Doleans-Dade formula that

$$
\begin{aligned}
L_{t} & =e^{B_{t}-B_{t}^{p}} \prod_{s \leq t}\left(1+F_{1}\left(X_{s-}, X_{s}\right)\right) e^{-F_{1}\left(X_{s-}, X_{s}\right)} \\
& =e^{-B_{t}^{p}} \prod_{s \leq t}\left(1+F_{1}\left(X_{s-}, X_{s}\right)\right) \\
& =e^{2 A_{\gamma F}(t)-B_{t}^{p}}
\end{aligned}
$$

is a non-negative local martingale under $\mathbf{P}_{x}$ for every $x \in E$. Hence $L_{t}$ is a supermartingale multiplicative functional of $X$. Thus

$$
\mathbf{E}_{x}\left[e^{2 A_{\gamma F}(t)-B_{t}^{p}}\right] \leq 1
$$

Applying the Cauchy-Schwartz inequality we get

$$
\begin{aligned}
\mathbf{E}_{x}\left[e_{\gamma F}(t)\right] & =\mathbf{E}_{x}\left[e^{A_{\gamma F}(t)-(1 / 2) B_{t}^{p}} e^{B_{t}^{p} / 2}\right] \\
& \leq\left(\mathbf{E}_{x}\left[e^{2 A_{2 \gamma F}(t)-B_{t}^{p}}\right]\right)^{1 / 2}\left(\mathbf{E}_{x}\left[e^{B_{t}^{p}}\right]\right)^{1 / 2} \\
& \leq\left(\mathbf{E}_{x}\left[e^{B_{t}^{p}}\right]\right)^{1 / 2} .
\end{aligned}
$$


As $\alpha q \in \mathbf{K}_{1}(X)$ and $B^{p} \in \mathbf{K}(X)$, it follows from Proposition 2.3 of [13] that there exists a $t_{0}>0$ such that

$$
\theta:=\max \left\{\sup _{x \in E} \mathbf{E}_{x}\left[A_{\alpha q}\left(t_{0}\right)\right], \sup _{x \in E} \mathbf{E}_{x}\left[B_{t_{0}}^{p}\right]\right\}<1 .
$$

Thus by Khasminskii's inequality and (2.3),

$$
\begin{aligned}
\sup _{x \in E} \mathbf{E}_{x}\left[e_{q+F}\left(t_{0}\right)\right] & \leq \sup _{x \in E}\left(\mathbf{E}_{x}\left[e_{\alpha q}\left(t_{0}\right)\right]\right)^{1 / \alpha}\left(\mathbf{E}_{x}\left[e_{\gamma F}\left(t_{0}\right)\right]\right)^{1 / \gamma} \\
& \leq\left(\frac{1}{1-\theta}\right)^{\frac{1}{\alpha}+\frac{1}{2 \gamma}}<\infty
\end{aligned}
$$

It follows from the above inequality and the Markov property of $X$ that there are constants $c_{1}, \beta_{1}>0$ such that

$$
\sup _{x \in E} \mathbf{E}_{x}\left[e_{q+F}(t)\right] \leq c_{1} e^{\beta_{1} t}
$$

The case of $q \in \mathbf{K}(X)$ and $F \in \mathbf{J}(X)$ can be handled using a similar but simpler argument, cf. the definition of $\mathbf{K}(X)$.

For $q \in \mathbf{K}_{1}(X) \cup \mathbf{K}(X)$ and $F \in \mathbf{J}(X)$, define a semigroup $\left\{T_{t}\right\}_{t \geq 0}$ by

$$
T_{t} f(x)=\mathbf{E}_{x}\left[e_{q+F}(t) f\left(X_{t}\right)\right], \quad f \geq 0 .
$$

For a measurable function $f$, let $\mu_{H} f$ denote the measure $f(x) \mu_{H}(d x)$. Recall that $\mu_{H}$ is the Revuz measure of $H$. We define a linear operator $\mathbf{F}$ that maps a bounded function $f$ on $E$ into the function

$$
\mathbf{F} f(x):=\int_{E_{\partial}}\left(e^{F(x, y)}-1\right) f(y) N(x, d y), \quad x \in E .
$$

When $\beta$ is greater than the constant $\beta_{1}$ in Proposition 2.3, the $\beta$-resolvent $V_{\beta}$ of the semigroup $T_{t}$

$$
V_{\beta} f(x):=\mathbf{E}_{x}\left[\int_{0}^{\zeta} e^{-\beta t} e_{q+F}(t) f\left(X_{t}\right) d t\right]
$$

is well defined on $E$ for any bounded function $f$ on $E$.

Theorem 2.4. For any $\beta$ greater than the constant $\beta_{1}$ in Proposition 2.3 and any bounded function $f$ on $E$, we have

$$
V_{\beta} f=G_{\beta} f+G_{\beta}\left(\left(\mu_{H} \mathbf{F}+q\right) V_{\beta} f\right) .
$$


Proof. For $s \leq t$, define $A_{s, t}:=\int_{s}^{t} q\left(X_{r}\right) d r+\sum_{s<r \leq t} F\left(X_{r-}, X_{r}\right)$, which is right continuous and has left limits as a function of $s$. Hence

$$
\begin{aligned}
e_{q+F}(t)-1 & =-\left(e^{A_{t, t}}-e^{A_{0, t}}\right) \\
& =-\left(\int_{0}^{t} e^{A_{s-, t}}\left(-q\left(X_{s}\right)\right) d s+\sum_{s \leq t}\left(e^{A_{s, t}}-e^{A_{s-, t}}\right)\right) \\
& =\int_{0}^{t} e^{A_{s, t}} q\left(X_{S}\right) d s+\sum_{s \leq t} e^{A_{s, t}}\left(e^{F\left(X_{s-}, X_{s}\right)}-1\right) .
\end{aligned}
$$

The last equality is due to the fact that $s \rightarrow X_{s}$ can only have at most countably many jumps over the time interval $[0, t]$ and hence $A_{s-, t}=A_{s, t}$ for all except countably many $s \in(0, t]$. For any $\beta$ larger than the constant $\beta_{1}$ in Proposition 2.3, we have

$$
\mathbf{E}_{x}\left[\int_{0}^{\zeta} e^{-\beta t} e_{q+F}(t) d t\right]<\infty .
$$

Thus by (2.6) and Fubini's theorem

$$
\begin{aligned}
V_{\beta} f(x)= & G_{\beta} f(x)+\mathbf{E}_{x}\left[\int_{0}^{\zeta} e^{-\beta t}\left(e_{q+F}(t)-1\right) f\left(X_{t}\right) d t\right] \\
= & G_{\beta} f(x)+\mathbf{E}_{x}\left[\int _ { 0 } ^ { \zeta } e ^ { - \beta t } \left(\int_{0}^{t} e^{A_{s, t}} q\left(X_{s}\right) d s\right.\right. \\
& \left.\left.+\sum_{s \leq t} e^{A_{s, t}}\left(e^{F\left(X_{s-}, X_{s}\right)}-1\right)\right) f\left(X_{t}\right) d t\right] \\
= & G_{\beta} f(x)+\mathbf{E}_{x}\left[\int_{0}^{\zeta} e^{-\beta s} q\left(X_{s}\right)\left(\int_{s}^{\zeta} e^{-\beta(t-s)} e^{A_{s, t}} f\left(X_{t}\right) d t\right) d s\right] \\
& +\mathbf{E}_{x}\left[\sum_{s \leq \zeta} e^{-\beta s}\left(e^{F\left(X_{s-}, X_{s}\right)}-1\right) \int_{s}^{\zeta} e^{-\beta(t-s)} e^{A_{s, t}} f\left(X_{t}\right) d t\right] \\
= & G_{\beta} f(x)+\mathbf{E}_{x}\left[\int_{0}^{\zeta} e^{-\beta s} q\left(X_{s}\right) V_{\beta} f\left(X_{s}\right) d s\right] \\
& +\mathbf{E}_{x}\left[\sum_{s \leq \zeta} e^{-\beta s}\left(e^{F\left(X_{s-}, X_{s}\right)}-1\right) V_{\beta} f\left(X_{s}\right)\right] \\
= & G_{\beta} f(x)+G_{\beta}\left(q V_{\beta} f\right)(x)+G_{\beta}\left(\mu_{H} \mathbf{F} V_{\beta} f\right)(x) \\
= & G_{\beta} f(x)+G_{\beta}\left(\left(\mu_{H} \mathbf{F}+q\right) V_{\beta} f\right)(x) .
\end{aligned}
$$

In the third to last equality we used the fact that $A_{s, t}=e_{q+F}(t-s) \circ \theta_{s}$ and the Markov property of $X$.

Remark 1. Let $\mathcal{L}$ be the infinitesimal generator of $X$. The above theorem suggests that the infinitesimal generator of the semigroup $T_{t}$ takes the form of $\mathcal{L}+\mu_{H} \mathbf{F}+q$. We will address the issue of $L^{2}$-generator of $T_{t}$ rigorously in section 4 of this paper under an extra condition that the bilinear form of $X$ satisfies a sector condition. 
Theorem 2.5. For every $x \in E$ with $g(x)<\infty, g\left(X_{t}\right)$ is right continuous in $t \in[0, \zeta)$ and has left limits in $t \in(0, \zeta) \mathbf{P}_{x}$-a.s. .

Proof. Let $x \in E$ be such that $g(x)<\infty$. By the strong Markov property of $X$, for any bounded stopping time $T$,

$g\left(X_{T}\right)=\mathbf{E}_{X_{T}}\left[e_{q+F}(\zeta)\right]=e_{-q-F}(T) \mathbf{E}_{x}\left[e_{q+F}(\zeta) \mid \mathcal{M}_{T}\right], \quad \mathbf{P}_{x}$-a.s. on $\{T<\zeta\}$

Here the martingale $t \rightarrow \mathbf{E}_{x}\left[e_{q+F}(\zeta) \mid \mathcal{M}_{t}\right]$ is taken to be the right continuous version. As $t \rightarrow X_{t}$ is right continuous with left limits and $g$ is Borel measurable, the process $t \rightarrow g\left(X_{t}\right)$ is optional. Hence by the Optional Section Theorem (cf. Theorem 4.10 in [27]), we have from (2.7) that

$$
\mathbf{P}_{x}\left(e_{q+F}(t) g\left(X_{t}\right)=\mathbf{E}_{x}\left[e_{q+F}(\zeta) \mid \mathcal{M}_{t}\right] \text { for all } t \in[0, \zeta)\right)=1 .
$$

Therefore $t \rightarrow g\left(X_{t}\right)$ is right continuous in $t \in[0, \zeta)$ and has left limits in $t \in(0, \zeta) \mathbf{P}_{x}$-a.s..

Theorem 2.6. Assume that $q \in \mathbf{K}_{1}(X)$ and $F \in \mathbf{J}_{\infty}(X)$. Then the gauge function $g$ is finely continuous. Furthermore the function $g$ is either bounded on $E$ or is identically $\infty$ on $E$.

Proof. Define $O=\{x \in E: g(x)<\infty\}$. Let $x \in O$ and $K$ be any closed subset of $E \backslash O$. Define $T_{K}=\inf \left\{t>0: X_{t} \in K\right\}$. By the strong Markov property,

$$
\infty>g(x) \geq \mathbf{E}_{x}\left[T_{K}<\zeta ; e_{q+F}\left(T_{K}\right) g\left(X_{T_{K}}\right)\right] .
$$

Since $K$ is closed, $X_{T_{K}} \in K$ by the right continuity of $t \rightarrow X_{t}$. Thus $g\left(X_{T_{K}}\right)=\infty$ on $\left\{T_{K}<\zeta\right\}$. On the other hand, $e_{q+F}\left(T_{K}\right)>0$ on $\left\{T_{K}<\zeta\right\} \mathbf{P}_{x}$-a.s.. It follows that $\mathbf{P}_{x}\left(T_{K}<\zeta\right)=0$. This being true for all closed subsets $K \subset E \backslash O$, we have

$$
\mathbf{P}_{x}\left(T_{O^{c}}<\zeta\right)=0 .
$$

Thus $O$ is absorbing.

Let $K, \delta$ and $\beta$ be specified as in Definition 2.1 for $q \in \mathbf{K}_{1}(X)$. Let $\alpha>1$ be such that $\alpha \beta<1$ and let $\gamma>1$ be such that $\frac{1}{\alpha}+\frac{1}{\gamma}=1$. Since $e^{2 \gamma F}-1$ is in $\mathbf{J}_{\infty}(X)$, there exist a set $K_{1} \supset K$ of finite $m$-measure and a $\delta_{1}<\delta$ such that

$$
\sup _{B \subset K_{1}: m(B)<\delta_{1}} \sup _{x \in E} \int_{K_{1}^{c} \cup B} G(x, y) \int_{E_{\partial}}\left|e^{2 \gamma F(y, z)}-1\right| N(y, d z) \mu_{H}(d y) \leq 1 / 2 .
$$

Choose $M$ large enough so that the set $\left\{x \in K_{1}: M<g(x)<\infty\right\}$ has $m$-measure less than $\delta_{1}$. Let $B=K_{1}^{c} \cup\left\{x \in K_{1}: M<g(x)<\infty\right\}$. Then by an argument similar to that used for deriving (2.4) we get

$$
\mathbf{E}_{x}\left[e_{q+F}\left(\tau_{B}\right)\right] \leq(1-\alpha \beta)^{-1 / \alpha} 2^{1 /(2 \gamma)}:=\eta,
$$

where $\tau_{B}:=T_{B^{c}}=\inf \left\{t>0: X_{t} \notin B\right\}$. Thus for $x \in E$, we have

$$
\begin{aligned}
g(x) & =\mathbf{E}_{x}\left[\tau_{B}=\zeta ; e_{q+F}\left(\tau_{B}\right)\right]+\mathbf{E}_{x}\left[\tau_{B}<\zeta ; e_{q+F}\left(\tau_{B}\right) g\left(X_{\tau_{B}}\right)\right] \\
& \leq \eta+\mathbf{E}_{x}\left[\tau_{B}<\zeta ; e_{q+F}\left(\tau_{B}\right) g\left(X_{\tau_{B}}\right)\right] .
\end{aligned}
$$


Note that for $x \in B \cap O, \mathbf{P}_{x}$-a.s. on $\left\{\tau_{B}<\zeta\right\}, X_{\tau_{B}}$ does not belong to $E \backslash O$ because $O$ is absorbing. So $g\left(X_{\tau_{B}}\right) \leq M$ as by Theorem $2.5 t \rightarrow g\left(X_{t}\right)$ is right continuous on $[0, \zeta)$. Therefore the second term on the right side of $(2.8)$ is bounded by $\eta M$. It follows that on $B \cap O, g$ is bounded by $\eta(1+M)$; it is bounded by $M$ on $O \backslash B$ by the definition of $B$. Thus $O=\{x \in E: g(x) \leq \eta(1+M)\}$.

We now show that the gauge function is finely continuous. It is equivalent to show that $t \rightarrow g\left(X_{t}\right)$ is right continuous on $[0, \zeta) \mathbf{P}_{x}$-a.s. for all $x \in E$. Define $T=\inf \left\{t>0: g\left(X_{t}\right)<\infty\right\}$ with the convention inf $\emptyset=\zeta$. Clearly $g\left(X_{t}\right)=\infty$ for $t<T$. It follows from Theorem 2.5 that $t \rightarrow g\left(X_{t}\right)$ is finite and right continuous for $t \in(T, \zeta) \mathbf{P}_{X}$-a.s. Hence it suffices to show that $g\left(X_{T}\right)<\infty \mathbf{P}_{X}$-a.s. on $\{T<\zeta\}$ and apply Theorem 2.5.

For this, observe that for each bounded stopping time $S$,

$$
\begin{aligned}
g\left(X_{S}\right) e_{q+F}(S) & =\mathbf{E}_{x}\left[e_{q+F}(\zeta) \mid \mathcal{M}_{S}\right] \\
& =\lim _{n \rightarrow \infty} \uparrow \mathbf{E}_{x}\left[e_{q+F}(\zeta) \wedge n \mid \mathcal{M}_{S}\right] \quad \mathbf{P}_{x} \text {-a.s. on }\{S<\zeta\},
\end{aligned}
$$

where the symbol $\uparrow$ indicates increasing convergence. Here the martingale

$$
t \rightarrow \mathbf{E}_{x}\left[e_{q+F}(\zeta) \wedge n \mid \mathcal{M}_{t}\right]
$$

is automatically taken to be the right continuous version. As $t \rightarrow X_{t}$ is right continuous with left limits and $g$ is Borel, so $t \rightarrow g\left(X_{t}\right)$ is optional. By the Optional Section Theorem again (cf. Theorem 4.10 of [27]), we have $\mathbf{P}_{x}$-a.s.

$$
g\left(X_{t}\right) e_{q+F}(t)=\lim _{n \rightarrow \infty} \uparrow \mathbf{E}_{x}\left[e_{q+F}(\zeta) \wedge n \mid \mathcal{M}_{t}\right] \quad \text { for all } t \in[0, \zeta) .
$$

On the other hand, $\mathbf{P}_{x}$-a.s. on $\{T<\zeta\}$, as $g\left(X_{T+s}\right) \leq \eta(1+M)$ for $s \in\left(0, \zeta \circ \theta_{T}\right)$, one has

$g\left(X_{T+s}\right) e_{q+F}(T+s) \leq \eta(1+M) e_{|q|+|F|}(T+1) \quad$ for all $0<s<1 \wedge\left(\zeta \circ \theta_{T}\right)$.

By (2.9) and the Optional Sampling theorem, $\mathbf{P}_{x}$-a.s. on $\{T<\zeta\}$,

$$
\mathbf{E}_{x}\left[e_{q+F}(\zeta) \wedge n \mid \mathcal{M}_{T+s}\right] \leq \eta(1+M) e_{|q|+|F|}(T+1)
$$

holds for every $n \geq 1$, all $s \in\left(0, \zeta \circ \theta_{T}\right)$ and hence for $s=0$ as well. Now by (2.9) again,

$$
g\left(X_{T}\right) e_{q+F}(T) \leq \eta(1+M) e_{|q|+|F|}(T+1) \quad \mathbf{P}_{x} \text {-a.s. on }\{T<\zeta\}
$$

In view of Proposition 2.2, this implies that $g\left(X_{T}\right)<\infty$. Now by Theorem 2.5,

$$
\lim _{r \downarrow T} g\left(X_{r}\right)=\lim _{s \downarrow 0} g\left(X_{s}\right) \circ \theta_{T}=g\left(X_{T}\right) \quad \mathbf{P}_{x} \text {-a.s. on }\{T<\zeta\} .
$$

This proves the fine continuity of $g$.

Since $O^{c}=\{x \in E: g(x)>\eta(1+M)\}$ is finely open, if $O^{c}$ is not empty then for $x \in O^{c}$,

$$
\int_{O^{c}} G(x, y) m(d y)=\mathbf{E}_{x}\left[\int_{0}^{\infty} 1_{O^{c}}\left(X_{s}\right) d s\right]>0
$$

and so $m\left(O^{c}\right)>0$. This would imply by the $m$-irreducibility of $X$ that $O$ can not be absorbing unless $O$ is empty. This says that either $O$ or $O^{c}$ is empty, and therefore either $g$ is identically infinity or bounded on $E$. 


\section{Conditional gauge}

In addition to the assumptions on $X$ made in Section 2, we assume that the process $X$ has a strong dual Borel standard process $\left(\widehat{X}, \widehat{\mathbf{P}}_{x}, x \in E\right)$ on $E$ with respect to the measure $m$. Under our assumption, $\widehat{G}(x, y)=G(y, x)$ is the Green function of the dual process $\widehat{X}$. Let $d:=\{(x, y) \in E \times E: G(x, y)=0$ or $G(x, y)=\infty\}$. For each fixed $z \in E$, set $E_{z}:=\{x \in E: 0<G(x, z)<\infty\}$

We are also going use $\left(\widehat{N}(x, d y), \widehat{H}_{t}\right)$, where $\widehat{N}(x, d y)$ is a Borel kernel on $\left(E, \mathcal{B}\left(E_{\partial}\right)\right)$ and $\widehat{H}_{t}$ is a positive continuous additive functional of $\widehat{X}$ with bounded 1-potential, to denote a Lévy system of $\widehat{X}$. According to [22], we may and do assume that $H_{t}$ and $\widehat{H}_{t}$ are dual additive functionals with Revuz measure $\mu_{H}$ and that $N$ and $\widehat{N}$ are dual kernels with respect to the measure $\mu_{H}$ on $E$, i. e.,

$$
\int_{E} \int_{E} f(x) g(y) N(x, d y) \mu_{H}(d x)=\int_{E} \int_{E} f(x) g(y) \widehat{N}(y, d x) \mu_{H}(d y)
$$

for all nonnegative functions $f$ and $g$ on $E$. That is, $N(x, d y) \mu_{H}(d x)=\widehat{N}(y, d x)$ $\mu_{H}(d y)$ on $E \times E$.

We first recall a definition from [13].

Definition 3.1. (1) A function $q$ is said to be in the class semi- $\mathbf{S}_{1}(X)$ if for each $z \in E$, there is a Borel set $K=K(z)$ of finite m-measure and a constant $\delta=$ $\delta(z)>0$ such that

$$
\beta_{1}(q):=\sup _{B \subset K: m(B)<\delta}\left(\sup _{x \in E_{z}} \int_{K^{c} \cup B} \frac{G(x, y) G(y, z)}{G(x, z)}|q(y)| m(d y)\right)<1 .
$$

(2) A function $q$ is said to be in class $\mathbf{S}_{1}(X)$ if there is a Borel set $K$ of finite $m$-measure and a constant $\delta>0$ such that

$$
\beta_{2}(q):=\sup _{B \subset K: m(B)<\delta}\left(\sup _{(x, z) \in(E \times E) \backslash d} \int_{K^{c} \cup B} \frac{G(x, y) G(y, z)}{G(x, z)}|q(y)| m(d y)\right)<1 .
$$

Now we introduce the following

Definition 3.2. Suppose $F$ is a Borel function on $E \times E$ vanishing on the diagonal.

(1) $F$ is said to be in the class semi- $\mathbf{A}_{\infty}(X)$ if $F$ is bounded and for any $\varepsilon>0$ and $w \in E$, there is a Borel subset $K=K(\varepsilon, w)$ of finite $m$-measure and a constant $\delta=\delta(\varepsilon, w)>0$ such that for all measurable set $B \subset K$ with $m(B)<\delta$,

$$
\sup _{x \in E_{w}} \int_{K^{c} \cup B} G(x, y)\left(\int_{E} \frac{|F(y, z)| G(z, w)}{G(x, w)} N(y, d z)\right) \mu_{H}(d y) \leq \varepsilon .
$$

(2) $F$ is said to be in the class $\mathbf{A}_{\infty}(X)$ if $F$ is bounded and for any $\varepsilon>0$ there is a Borel subset $K=K(\varepsilon)$ of finite $m$-measure and a constant $\delta=\delta(\varepsilon)>0$ such that for all measurable set $B \subset K$ with $m(B)<\delta$,

$$
\sup _{(x, w) \in(E \times E) \backslash d} \int_{(K \backslash B)^{c} \times(K \backslash B)^{c}} G(x, y) \frac{|F(y, z)| G(z, w)}{G(x, w)} N(y, d z) \mu_{H}(d y) \leq \varepsilon .
$$


It is easy to see that if $F_{1}$ and $F_{2}$ belong to semi- $\mathbf{A}_{\infty}(X)$ (respectively $\mathbf{A}_{\infty}(X)$ ) and $c$ is a constant, then $c F_{1}, F_{1}+F_{2}, F_{1} F_{2}$ all belong to semi- $\mathbf{A}_{\infty}(X)$ (respectively $\left.\mathbf{A}_{\infty}(X)\right)$. Furthermore, one can also show easily that if $F$ belongs to semi- $\mathbf{A}_{\infty}(X)$ (respectively $\mathbf{A}_{\infty}(X)$ ), then the function $e^{F}-1$ belongs to semi- $\mathbf{A}_{\infty}(X)$ (respectively $\left.\mathbf{A}_{\infty}(X)\right)$.

Clearly $\mathbf{A}_{\infty}(X) \subset$ semi- $\mathbf{A}_{\infty}(X)$; and $F \in \mathbf{A}_{\infty}(X)$ if and only if $\widehat{F} \in \mathbf{A}_{\infty}(\widehat{X})$, where $\widehat{F}(x, y):=F(y, x)$. It is shown in Corollary 3.1 of [13] that $\mathbf{S}_{1}(X) \subset \mathbf{K}_{1}(X)$. Concrete examples of $\mathbf{S}_{1}(X)$ functions were also given in [13]. Here we present some examples of functions in $\mathbf{A}_{\infty}(X)$.

Example 1 . In the case when $X$ a killed symmetric $\alpha$-stable process on a bounded $\kappa$-fat set $D$ of $\mathbf{R}^{n}$ for some $\alpha \in(0,2), \kappa \in(0,1)$ and $n \geq 2$, it is well known that $H_{t}=\widehat{H}_{t}=t, N(x, d y)=\mathcal{A}(n,-\alpha)|x-y|^{-n-\alpha} d y=\widehat{N}(x, d y)$ on $D$, $N(x, \partial)=\mathcal{A}(n,-\alpha) \int_{D^{c}}|x-y|^{-n-\alpha}=\widehat{N}(x, \partial)$, where

$$
\mathcal{A}(n,-\alpha)=\frac{\alpha \Gamma\left(\frac{n+\alpha}{2}\right)}{2^{1-\alpha} \pi^{n / 2} \Gamma\left(1-\frac{\alpha}{2}\right)} .
$$

It follows easily from the $3 G$ inequality (see [41]) that if a function $F$ on $D \times D$ satisfies the inequality

$$
|F(x, y)| \leq C|x-y|^{n+\alpha} G_{D}(x, y)
$$

for some $C>0$, then $F \in \mathbf{A}_{\infty}(X)$.

Example 2. Suppose that $X$ is a killed symmetric $\alpha$-stable process on a bounded $C^{1,1}$ domain $D$ of $\mathbf{R}^{n}$ for some $\alpha \in(0,2)$. If $F$ is a function on $D \times D$ satisfying the inequality

$$
|F(x, y)| \leq C|x-y|^{\beta}, \quad x, y \in D
$$

for some constants $\beta>\alpha$ and $C>0$, then $F \in \mathbf{A}_{\infty}(X)$. In fact, it is shown in Theorems 1.1 and 1.2 of [10] (cf. also [31]) that there exist constants $C_{1}=C_{1}(D, \alpha)>0$ and $C_{2}=C_{2}(D, \alpha)>0$ such that

$$
\begin{aligned}
& G_{D}(x, y) \leq C_{1} \min \left\{\frac{1}{|x-y|^{n-\alpha}}, \frac{\delta_{D}(x)^{\alpha / 2}}{|x-y|^{n-\alpha / 2}}\right\}, \\
& G_{D}(x, w) \geq C_{2} \frac{1}{|x-w|^{n-\alpha}}, \quad \text { when }|x-w| \leq \frac{1}{2} \max \left\{\delta_{D}(x), \delta_{D}(w)\right\}, \\
& G_{D}(x, w) \geq C_{2} \frac{\delta_{D}(x)^{\alpha / 2} \delta_{D}(w)^{\alpha / 2}}{|x-w|^{n}}, \quad \text { when }|x-w| \geq \frac{1}{2} \max \left\{\delta_{D}(x), \delta_{D}(w)\right\},
\end{aligned}
$$

where $\delta_{D}(x)$ the Euclidean distance between $x$ and $D^{c}$. It follows that there exists a constant $C_{3}=C_{3}(D, \alpha)$ such that when $|x-w| \leq \frac{1}{2} \max \left\{\delta_{D}(x), \delta_{D}(w)\right\}$,

$$
\frac{G_{D}(x, y) G_{D}(z, w)}{G_{D}(x, w)} \leq C_{3} \frac{|x-w|^{n-\alpha}}{|x-y|^{n-\alpha}|z-w|^{n-\alpha}}
$$

and when $|x-w| \geq \max \left\{\delta_{D}(x), \delta_{D}(w)\right\}$,

$$
\frac{G_{D}(x, y) G_{D}(z, w)}{G_{D}(x, w)} \leq C_{3} \frac{|x-w|^{n-\alpha / 2}}{|x-y|^{n-\alpha / 2}|z-w|^{n-\alpha / 2}} .
$$


Hence we have

$$
\begin{aligned}
& \frac{G_{D}(x, y)|F(y, z)| G_{D}(z, w)}{G_{D}(x, w)|y-z|^{n+\alpha}} \\
& \leq C\left(\frac{|x-w|^{n-\alpha / 2}}{|x-y|^{n-\alpha / 2}|z-w|^{n-\alpha / 2}|y-z|^{n+\alpha-\beta}}+\frac{|x-w|^{n-\alpha}}{|x-y|^{n-\alpha}|z-w|^{n-\alpha}|y-z|^{n+\alpha-\beta}}\right) \\
& \leq C \cdot C_{3}\left(\frac{1}{|z-w|^{n-\alpha / 2}|y-z|^{n+\alpha-\beta}}+\frac{1}{|x-y|^{n-\alpha / 2}|y-z|^{n+\alpha-\beta}}\right. \\
& +\frac{1}{|z-w|^{n-\alpha}|y-z|^{n+\alpha-\beta}}+\frac{1}{|x-y|^{n-\alpha}|y-z|^{n+\alpha-\beta}} \\
& \left.+\frac{1}{|x-y|^{n-\alpha / 2}|z-w|^{n-\alpha / 2}|y-z|^{3 \alpha / 2-\beta}}+\frac{1}{|x-y|^{n-\alpha}|z-w|^{n-\alpha}|y-z|^{2 \alpha-\beta}}\right)
\end{aligned}
$$

Therefore to show that $F \in \mathbf{A}_{\infty}(X)$, we only need to show that for any $\varepsilon>0$, there is a $\delta=\delta(\varepsilon)>0$ such that for any Borel set $B \subset D$ with $m(B)<\delta$ we have

$$
\begin{aligned}
& \sup _{x, w \in D} \int_{B} d y \int_{D} \frac{1}{|x-y|^{n-\alpha / 2}|z-w|^{n-\alpha / 2}|y-z|^{3 \alpha / 2-\beta}} d z \leq \varepsilon \\
& \sup _{x, w \in D} \int_{B} d y \int_{D} \frac{1}{|x-y|^{n-\alpha}|z-w|^{n-\alpha}|y-z|^{2 \alpha-\beta}} d z \leq \varepsilon .
\end{aligned}
$$

By Hölder's inequality,

$$
\begin{aligned}
& \sup _{y, w \in D} \int_{D} \frac{1}{|z-w|^{n-\alpha / 2}|y-z|^{3 \alpha / 2-\beta}} d z<\infty, \\
& \sup _{y, w \in D} \int_{D} \frac{1}{|z-w|^{n-\alpha}|y-z|^{2 \alpha-\beta}} d z<\infty,
\end{aligned}
$$

Now (3.4) and (3.5) are easy consequences of the two inequalities above.

Proposition 3.1. Suppose that $F$ is a bounded Borel function on $E \times E$ vanishing on the diagonal. Then $F$ is in $\mathbf{A}_{\infty}(X)$ if and only if for every $\varepsilon>0$ there is a subset $K=K(\varepsilon)$ and a constant $\delta=\delta(\varepsilon)>0$ such that for any excessive function $f$ of $X$ and all measurable set $B \subset K$ with $m(B)<\delta$,

$$
\int_{(K \backslash B)^{c} \times(K \backslash B)^{c}} G(x, y)|F(y, z)| f(z) N(y, d z) \mu_{H}(d y) \leq \varepsilon f(x) \text { for all } x \in E .
$$

Proof. For any $z \in E$, the functions $y \rightarrow G(y, z)$ is an excessive function of $X$. So (3.6) implies (3.1) and therefore $F$ is in $\mathbf{A}_{\infty}(X)$ if the condition (3.6) is satisfied. Conversely suppose that (3.1) holds. Then (3.6) is valid when $f$ is the potential of some measure $v$. Now the conclusion follows because any excessive function is the increasing limit of a sequence of potentials of the form $G h_{n}$, where $h_{n}=n\left(f-n G_{n} f\right) \geq 0$.

Since the constant function 1 is excessive with respect to $X$, we can take $f=1$ in the Proposition above and get 
Corollary 3.2. $\mathbf{A}_{\infty}(X) \subset \mathbf{J}_{\infty}(X)$.

For each $z \in E$, let $E_{z}:=\{x \in E: 0<G(x, z)<\infty\}$ and $X^{\cdot}, z=\left(X, \mathbf{P}_{x}^{z}, x \in\right.$ $E_{z}$ ) be the $h$-conditioned process of $X$ with $h(\cdot)=G(\cdot, z)$; that is, $X^{\cdot, z}$ has transition probability $q(t, x, d y)=p(t, x, d y) G(y, z) / G(x, z)$. As we previously mentioned that, by [32] or Proposition 2.2 of [16], $X^{\cdot, z}$ is a Borel standard process on $E_{z}$. It follows from Proposition 5.4, Theorem 6.5 and in particular Example 6.14 in [25] that $X^{\cdot, z}$ is a transient with finite lifetime $\zeta^{z}$ and $\lim _{t \uparrow \zeta^{z}} X_{t}^{\cdot, z}=z$ almost surely. Clearly, the conditional process $X^{\cdot}, z$ is irreducible. Note that the Green function for $X^{\cdot}, z$ with respect to $m$ is

$$
\frac{G(x, \cdot) G(\cdot, z)}{G(x, z)}
$$

The following result gives a Lévy system for the conditioned process $X^{\cdot}, w$.

Proposition 3.3. For any non-negative Borel function $f$ on $E \times E$ vanishing on diagonal and any $x, w \in E$ with $0<G(x, w)<\infty$,

$$
\mathbf{E}_{x}^{w}\left[\sum_{s \leq t} f\left(X_{s-}, X_{s}\right)\right]=\mathbf{E}_{x}\left[\int_{0}^{t} \int_{E} \frac{f\left(X_{s}, y\right) G(y, w)}{G(x, w)} N\left(X_{s}, d y\right) d H_{s}\right] .
$$

Furthermore, a Lévy system of the conditioned process $X^{\cdot, w}$ is given by $\left(N^{w}, H_{t}\right)$ where

$$
N^{w}(x, d y)=\frac{G(y, w) N(x, d y)}{G(x, w)} .
$$

Proof. Let

$$
Z_{s}=\frac{G\left(X_{s}, w\right)}{G\left(X_{0}, w\right)}
$$

Then $Z_{s}$ is a supermartingale multiplicative functional of $X$. It follows from Section 62 of [38] that

$$
\begin{aligned}
\mathbf{E}_{x}^{w}\left[\sum_{s \leq t} f\left(X_{s-}, X_{s}\right)\right] \\
=\mathbf{E}_{x}\left[\sum_{s \leq t} f\left(X_{s-}, X_{s}\right)\left(\int_{0}^{\zeta} 1_{\{s<r \leq \zeta\}} d\left(-Z_{r}\right)+Z_{\zeta}\right) ; t<\zeta\right] \\
=\mathbf{E}_{x}\left[\sum_{s \leq t} f\left(X_{s-}, X_{s}\right) Z_{s} ; t<\zeta\right] \\
=\frac{1}{G(x, w)} \mathbf{E}_{x}\left[\sum_{s \leq t} f\left(X_{s-}, X_{s}\right) G\left(X_{s}, w\right) ; t<\zeta\right] \\
=\mathbf{E}_{x}\left[\int_{0}^{t} \int_{E} \frac{f\left(X_{s}, y\right) G(y, w)}{G(x, w)} N\left(X_{s}, d y\right) d H_{s}\right]
\end{aligned}
$$


Similarly, we have

$$
\begin{aligned}
\mathbf{E}_{x}^{w}\left[\int_{0}^{t} \int_{E} f\left(X_{s}, y\right) N^{w}\left(X_{s}, d y\right) d H_{s}\right] \\
=\mathbf{E}_{x}^{w}\left[\int_{0}^{t} \int_{E} \frac{f\left(X_{s}, y\right) G(y, w)}{G\left(X_{s}, w\right)} N\left(X_{s}, d y\right) d H_{s}\right] \\
=\mathbf{E}_{x}\left[\int_{0}^{t} d H_{s} \int_{E} \frac{f\left(X_{s}, y\right) G(y, w)}{G\left(X_{s}, w\right)} N\left(X_{s}, d y\right)\right. \\
=\mathbf{E}_{x}\left[\int_{0}^{t} d H_{s} \int_{E}^{\zeta} \frac{f\left(X_{s}, y\right) G(y, w)}{G\left(X_{s}, w\right)} N\left(X_{s}, d y\right) Z_{s} ; t<\zeta\right] \\
=\mathbf{E}_{x}\left[\int_{0}^{t} \int_{E} \frac{f\left(X_{s}, y\right) G(y, w)}{G(x, w)} N\left(X_{s}, d y\right) d H_{s}\right] .
\end{aligned}
$$

So the second conclusion of the proposition is valid.

Remark 2. It follows from the Proposition above that (cf. the proof of Proposition 2.2) for $q \in \mathbf{S}_{1}(X)$ and $F \in \mathbf{A}_{\infty}(X)$, the function

$$
\begin{aligned}
(x, w) \mapsto \mathbf{E}_{x}^{w}\left[A_{|q|+|F|}\left(\zeta^{w}\right)\right]= & \int_{E} \frac{G(x, y)|q(y)| G(y, w)}{G(x, w)} m(d y) \\
& +\int_{E} G(x, y)\left(\int_{E} \frac{|F(y, z)| G(z, w)}{G(x, w)} N(y, d z)\right) \\
& \times \mu_{H}(d y)
\end{aligned}
$$

is bounded on $(E \times E) \backslash d$, where $d=\{(x, y) \in E \times E: G(x, y)=0$ or $G(x, y)=$ $\infty$. Hence $e_{q+F}\left(\zeta^{w}\right)$ is well defined and by Jensen's inequality,

$$
\inf _{(x, w) \in(E \times E) \backslash d} \mathbf{E}_{x}^{w}\left[e_{q+F}\left(\zeta^{w}\right)\right]>0 .
$$

Theorem 3.4. Suppose that $q$ and $F$ are in the classes semi- $\mathbf{S}_{1}(X)$ and semi$\mathbf{A}_{\infty}(X)$, respectively. Then for each $z \in E$, either $\mathbf{E}_{x}^{z}\left[e_{q+F}\left(\zeta^{z}\right)\right] \equiv \infty$ or $x \rightarrow$ $\mathbf{E}_{x}^{z}\left[e_{q+F}\left(\zeta^{z}\right)\right]$ is bounded on $E_{z}$.

Proof. By Proposition 3.3, we have semi-S $\mathbf{S}_{\infty}(X)=\cap_{z \in E} \mathbf{K}_{1}\left(X^{\cdot}, z\right)$ and semi- $\mathbf{A}_{\infty}$ $(X)=\cap_{z \in E} \mathbf{J}_{\infty}\left(X^{\cdot}, z\right)$. So the theorem follows immediately from Theorem 2.6.

It follows from Theorem 6.5 and Example 6.14 of [25] that for fixed $x, z \in E$ with $0<G(x, z)<\infty$, the time reversal at the lifetime of the process $\left(X, \mathbf{P}_{x}^{z}\right)$ (obtained by reversing the conditional process $\left(X, \mathbf{P}_{x}^{z}\right)$ at its lifetime $\zeta^{z}$ and taking the right continuous version) has the same distribution as the conditioned process $\left(\widehat{X}, \widehat{\mathbf{P}}_{z}^{x}\right)$. Consequently

$$
\mathbf{E}_{x}^{z}\left[e_{q+F}\left(\zeta^{z}\right)\right]=\widehat{\mathbf{E}}_{z}^{x}\left[e_{q+\widehat{F}}\left(\widehat{\zeta}^{x}\right)\right]
$$

where $\widehat{F}$ is defined by $\widehat{F}(y, w)=F(w, y)$. 
Theorem 3.5. Suppose that $q \in \mathbf{S}_{1}(X)$ and $F \in \mathbf{A}_{\infty}(X)$. If $\mathbf{E}_{x}^{w}\left[e_{q+F}\left(\zeta^{w}\right)\right]$ is finite for some $\left(x_{0}, w_{0}\right) \in(E \times E) \backslash d$, then $\mathbf{E}_{x}^{w}\left[e_{q+F}\left(\zeta^{w}\right)\right]$ is bounded on $(E \times E) \backslash d$.

Proof. Let $u(x, w)=\mathbf{E}_{x}^{w}\left[e_{q+F}\left(\zeta^{w}\right)\right]$ and $\widehat{u}(x, w)=\widehat{\mathbf{E}}_{x}^{w}\left[e_{q+\widehat{F}}\left(\widehat{\zeta}^{w}\right)\right]$ for $(x, w) \notin$ $d$. Applying Theorem 3.4 to the process $X$ and using (3.7), we get

$$
\sup _{x \in E_{w_{0}}} \widehat{u}\left(w_{0}, x\right)=\sup _{x \in E_{w_{0}}} u\left(x, w_{0}\right)<\infty
$$

This implies by applying Theorem 3.4 to the process $\widehat{X}$ that for any $x \in E$,

$$
\sup _{z \in \widehat{E}_{x}} u(x, z)=\sup _{z \in \widehat{E}_{x}} \widehat{u}(z, x)<\infty \text {. }
$$

where $\widehat{E}_{x}=\{z \in E: 0<G(z, x)<\infty\}$.

Let $K, \delta$ and $\beta_{2}$ be specified as in Definition 3.1 for $q \in \mathbf{S}_{1}(X)$. Let $\alpha>1$ be such that $\alpha \beta_{2}<1$ and let $\gamma>1$ be such that $\frac{1}{\alpha}+\frac{1}{\gamma}=1$. Since $e^{2 \gamma F}-1$ is in $\mathbf{A}_{\infty}(X)$, there exist a set $K_{1} \supset K$ of finite $m$-measure and a $\delta_{1}<\delta$ such that for every $B \subset K_{1}$ with $m(B)<\delta_{1}$

$$
\sup _{x, w \in E} \int_{\left(K_{1} \backslash B\right)^{c} \times\left(K_{1} \backslash B\right)^{c}} \frac{G(x, y)\left|e^{2 \gamma F(y, z)}-1\right| G(z, w)}{G(x, w)} N(y, d z) \mu_{H}(d y) \leq \frac{1}{2} .
$$

Note that $u(x, z)$ is $\mathcal{B}(E \times E)$ if we set $u(x, z)=1$ for $(x, z) \in d$. Hence $\{x \in$ $\left.K_{1}: \sup _{z \in E} u(x, z)>M\right\}$ is $\mathcal{B}(E)$-measurable as it is the $x$-projection of the set $\left\{(x, z) \in K_{1} \times E: u(x, z)>M\right\}$. As $\bigcap_{M=2}^{\infty}\left\{x \in K_{1}: \sup _{z \in E} u(x, z)>M\right\}=\emptyset$, we can choose $M$ large enough so that the set $\left\{x \in K_{1}: \sup _{w \in E} u(x, w)>M\right\}$ has $m$ measure less than $\delta_{1}$. Let $B=K_{1}^{c} \cup\left\{x \in K_{1}: \sup _{z \in E} u(x, w)>M\right\}$. Similar to the argument leading to (2.4), we can show that for any $(x, w) \in(E \times E) \backslash d$,

$$
\mathbf{E}_{x}^{w}\left[e_{F}\left(\tau_{B}\right)\right] \leq\left(1-\alpha \beta_{2}\right)^{-1 / \alpha} 2^{1 /(2 \gamma)}:=\eta<\infty .
$$

Thus for any $(x, w) \in(E \times E) \backslash d$,

$$
\begin{aligned}
u(x, w) & =\mathbf{E}_{x}^{w}\left[\tau_{B}=\zeta^{w} ; e_{q+F}\left(\tau_{B}\right)\right]+\mathbf{E}_{x}^{w}\left[\tau_{B}<\zeta^{w} ; e_{q+F}\left(\tau_{B}\right) u\left(X_{\tau_{B}}^{\cdot, w}, w\right)\right] \\
& \leq \eta+\mathbf{E}_{x}^{w}\left[\tau_{B}<\zeta^{w} ; e_{q+F}\left(\tau_{B}\right) u\left(X_{\tau_{B}}^{\cdot, w}, w\right)\right]
\end{aligned}
$$

Observe also that for $x \in B \cap E_{w}, \mathbf{P}_{x}^{w}$-a.s. on $\left\{\tau_{B}\left(X^{\cdot}, w\right)<\zeta^{w}\right\}, u\left(X_{\tau_{B}}^{\cdot, w}, w\right) \leq M$ since by Theorem $2.5 t \rightarrow u\left(X_{t}^{\cdot}, w, w\right)$ is right continuous on $\left[0, \zeta^{w}\right)$. Therefore the second term on the right side of (3.8) is bounded by $\eta M$. It follows that for $x \in B \cap E_{w}, u(x, w) \leq \eta(1+M)$. By the definition of $B$ we know that for $x \in B^{c} \cap E_{w}, u(x, w) \leq M$. Hence for any $w \in E, \sup _{x \in E_{w}} u(x, w) \leq \eta(1+M)$. 
Recall that $\mathcal{L}$ is the infinitesimal generator of $X, \mu_{H}$ is the Revuz measure of $H_{t}$, and $\mathbf{F}$ is the operator that maps any bounded function $f$ on $E$ into the function

$$
\mathbf{F} f(x):=\int_{E}\left(e^{F(x, y)}-1\right) f(y) N(x, d y) .
$$

Theorem 3.6. Let $q \in \mathbf{S}_{1}(X)$ and $F \in \mathbf{A}_{\infty}(X)$ be such that the conditional gauge function $u(x, y)=\mathbf{E}_{x}^{y}\left[e_{q+F}\left(\zeta^{y}\right)\right]$ is not identically infinity on $(E \times E) \backslash d$. Define $V(x, y)=u(x, y) G(x, y)$ for $(x, y) \notin d$ and define $V f(x)=\int_{E} V(x, y) f(x, y)$ $m(d y)$ whenever it exists. Then for any function $\phi$ with $G|\phi|$ bounded,

$$
V \phi=G \phi+G\left(\left(q+\mu_{H} \mathbf{F}\right)(V \phi)\right) .
$$

Proof. From Theorem 3.5 we know that under the assumptions of the theorem, $u(x, y)$ is bounded for $(x, y) \in(E \times E) \backslash d$. Hence

$$
\begin{aligned}
\sup _{(x, y) \in(E \times E) \backslash d} \int_{E} G(x, w) & \left(\int_{E} \frac{\left|e^{F(w, z)}-1\right| u(z, y) G(z, y)}{G(x, y)} N(w, d z)\right) \\
& \times \mu_{H}(d w)<\infty .
\end{aligned}
$$

By (2.6), the Markov property of $X$ and Proposition 3.3, we have

$$
\begin{aligned}
\mathbf{E}_{x}^{y}\left[e_{q+F}\left(\zeta^{y}\right)\right]-1 \\
=\mathbf{E}_{x}^{y}\left[\int_{0}^{\zeta^{y}} e_{q+F}\left(\zeta^{y}\right) \circ \theta_{s} q\left(X_{s}\right) d s+\sum_{s \leq \zeta^{y}} e_{q+F}\left(\zeta^{y}\right) \circ \theta_{s}\left(e^{F\left(X_{s-}, X_{s}\right)}-1\right)\right] \\
=\mathbf{E}_{x}^{y}\left[\int_{0}^{\zeta^{y}} u\left(X_{s}, y\right) q\left(X_{s}\right) d s+\sum_{s \leq \zeta^{y}} u\left(X_{s}, y\right)\left(e^{F\left(X_{s-}, X_{s}\right)}-1\right)\right] \\
=G(x, y)^{-1} \int_{E} G(x, z) u(z, y) q(z) G(z, y) m(d z) \\
\quad+G(x, y)^{-1} \int_{E} G(x, w)\left(\int_{E}\left(e^{F(w, z)}-1\right) u(z, y) G(z, y) N(w, d z)\right) \mu_{H}(d w) .
\end{aligned}
$$

Consequently

$$
\begin{aligned}
V(x, y)= & G(x, y)+\int_{E} G(x, z) q(z) V(z, y) m(d z) \\
& +\int_{E} G(x, w)\left(\int_{E}\left(e^{F(w, z)}-1\right) V(z, y) N(w, d z)\right) \mu_{H}(d w) .
\end{aligned}
$$

Thus we have

$$
V \phi(x)=G \phi(x)+G(q(V \phi))(x)+G\left(\mu_{H} \mathbf{F}(V \phi)\right)(x) .
$$


Remark 3. The above result together with Theorem 2.4 suggests that $V(x, y)$ is the Green function for Schrödinger semigroup $\left.\left\{T_{t}, t \geq 0\right]\right\}$ defined in (2.5). Recently it is shown in [8] that $V(x, y)$ is indeed the Green function for $\left\{T_{t}, t \geq 0\right\}$. More precisely, it is shown in [8] that for any nonnegative Borel function $f$ on $E$,

$$
\int_{E} V(x, y) f(y) m(d y)=\mathbf{E}_{x}\left[\int_{0}^{\zeta} e_{q+F}(t) f\left(X_{t}\right) d t\right] .
$$

\section{Generator of the Schrödinger semigroup}

In this section, $X$ is an irreducible transient Borel standard process on a Lusin space $E$ with Green function $G(x, y)$, as is specified at the beginning of Section 1. In Theorem 2.4, we gave a formula for the generator of the Schrödinger semigroup $T_{t}$ defined in (2.5). But what is the domain of this generator? We will answer this question precisely under an assumption that the Dirichlet form $(\mathcal{E}, \mathcal{F})$ of $X$ is nearly symmetric, that is, it satisfies Silverstein's sector condition:

there is $K \geq 1$ such that $\mathcal{E}_{1}(u, v)^{2} \leq K \mathcal{E}_{1}(u, u) \mathcal{E}_{1}(v, v) \quad$ for all $u, v \in \mathcal{F}$.

Here for $\beta>0, \mathcal{E}_{\beta}:=\mathcal{E}+\beta(\cdot, \cdot)_{L^{2}(E, m)}$. It is easy to see that if (4.1) holds then it holds with $\mathcal{E}_{\beta}$ in place of $\mathcal{E}_{1}$ for every $\beta \geq 1$. It is known from the results of [3], [9] and [17] that such a process $X$ is quasi-homeomorphic to a nearly $m$-symmetric irreducible transient Hunt process with a dual Hunt process on a locally compact separable metric space whose Dirichlet form is regular. Without loss of generality, we may and do assume in the following that $X$ is a nearly symmetric irreducible transient Hunt process on a locally compact separable metric space $E$ whose Dirichlet form $(\mathcal{E}, \mathcal{F})$ is regular. Note that $X$ has a dual Hunt process $\widehat{X}$ whose Dirichlet form is $(\widehat{\mathcal{E}}, \mathcal{F})$, where $\widehat{\mathcal{E}}(u, v)=\mathcal{E}(v, u)$ for $u, v \in \mathcal{F}$. Let $\overline{\mathcal{E}}=\frac{1}{2}(\mathcal{E}+\widehat{\mathcal{E}})$. It is well known that $(\overline{\mathcal{E}}, \mathcal{F})$ is a regular Dirichlet form in $L^{2}(E, m)$. We refer the readers to [21], [35], and [33] for the definitions and basics of Dirichlet forms, such as capacity, quasi-continuous version, measure of finite energy integral, smooth measure. For each $u \in \mathcal{F}$, it is well known that it has a quasi-continuous version. It is understood throughout this section that functions in $\mathcal{F}$ are represented by their quasi-continuous versions.

Under the above assumptions, the Green function $G(x, y)$ can be uniquely chosen so that for each $y \in E, x \mapsto G(x, y)$ is excessive for $X$ and for each $x$, $y \mapsto G(x, y)$ is excessive for $\widehat{X}$. We can therefore extend the Kato class $\mathbf{K}(X)$ and $\mathbf{K}_{1}(X)$ to smooth measures that may not be absolutely continuous with respect to the reference measure $m$. For a signed smooth measure $v$, let $A^{v}$ and $\left|A^{v}\right|$ be the continuous additive functionals having Revuz measures $v$ and $|v|$ respectively. Extending Definition 2.1(4), we say throughout this section that a signed smooth measure $v$ is in $\mathbf{K}(X)$ if

$$
\lim _{t \rightarrow 0} \sup _{x \in E} \mathbf{E}_{x}\left[\left|A^{v}\right|_{t}\right]=0 ;
$$

and $v \in \mathbf{K}_{1}(X)$ if (2.2) is satisfied with $|\nu|(d y)$ in place of $|q(y)| m(d y)$ and $\beta(v)$ in place of $\beta(q)$. 
Proposition 4.1. For $u \in \mathcal{F}$ and $\beta \geq 1$,

$$
\int_{0}^{\infty} t \widehat{\operatorname{Cap}}_{(\beta)}(\{|u| \geq t\}) d t \leq 2 K \mathcal{E}_{\beta}(u, u),
$$

where $\widehat{\operatorname{Cap}}_{(\beta)}$ stands for the $\beta$-capacity of $\widehat{X}$ and $K$ is the constant in the sector condition (4.1).

Proof. For each fixed $t>0$, let $K_{t}:=\{x \in E:|u| \geq t\}$ and

$$
\mathcal{F}_{K_{t}}:=\inf \left\{v \in \mathcal{F}: v \geq 1 \text { q.e. on } K_{t}\right\} \text {. }
$$

Clearly $\mathcal{F}_{K_{t}}$ is non-empty. It is known (cf. [21], [29], [35] and [33]) that there is a unique $\beta$-order equilibrium co-potential $\widehat{e}_{K_{t}} \in \mathcal{F}_{K_{t}}$ of $K_{t}$ and a unique symmetric $\beta$-order equilibrium potential $\bar{e}_{K_{t}} \in \mathcal{F}_{K_{t}}$ for $K_{t}$. Particularly, $\mathcal{E}_{\beta}\left(\widehat{e}_{K_{t}}, \widehat{e}_{K_{t}}\right)=$ $\widehat{\operatorname{Cap}}_{(\beta)}\left(K_{t}\right)$, and $\bar{e}_{K_{t}}$ is the unique $\overline{\mathcal{E}}_{\beta}$-minimizer in $\mathcal{F}_{K_{t}}$ with $\mathcal{E}_{\beta}\left(\bar{e}_{K_{t}}, \bar{e}_{K_{t}}\right)=$ $\overline{\operatorname{Cap}}_{(\beta)}\left(K_{t}\right)$, the $\beta$-capacity of $K_{t}$ with respect to the symmetric Dirichlet form $(\overline{\mathcal{E}}, \mathcal{F})$. By Lemma III.1.4 of [33], and (4.1) above

$$
\widehat{\operatorname{Cap}}_{(\beta)}\left(K_{t}\right)=\mathcal{E}_{\beta}\left(\bar{e}_{K_{t}}, \widehat{e}_{K_{t}}\right) \leq \sqrt{K} \mathcal{E}_{\beta}\left(\bar{e}_{K_{t}}, \bar{e}_{K_{t}}\right)^{1 / 2} \mathcal{E}_{\beta}\left(\widehat{e}_{K_{t}}, \widehat{e}_{K_{t}}\right)^{1 / 2}
$$

so

$$
\widehat{\operatorname{Cap}}_{(\beta)}\left(K_{t}\right) \leq K \overline{\operatorname{Cap}}_{(\beta)}\left(K_{t}\right)
$$

As Proposition 2 of [44] tells us

$$
\int_{0}^{\infty} t \overline{\operatorname{Cap}}_{(\beta)}\left(K_{t}\right) d t \leq 2 K \mathcal{E}_{(\beta)}(u, u),
$$

the assertion of our proposition is valid in view of (4.2).

Remark 4. The earliest inequality of the type given in this proposition may be due to Maz'ya for Sobolev spaces, see [34]. For results of this type in the case of symmetric processes, see [26], [30] and [44]. In [36] Rao established the inequality in Proposition 4.1 for functions $u=G_{\beta} f$, where $f \in L^{2}(E, m)$, with the constant 4 in place of $2 K$.

Proposition 4.2. Let $v$ be a positive smooth measure. Then for any $u \in \mathcal{F}$ and $\beta \geq 0$,

$$
\int_{E} u^{2}(x) v(d x) \leq 4 K\left\|G_{\beta} v\right\|_{\infty} \mathcal{E}_{\beta}(u, u),
$$

where $K \geq 1$ is the constant in (4.1).

Proof. Note that for positive smooth measures $v$ and $\eta$,

$$
\int_{E} \widehat{G}_{\beta} \eta(x) v(d x)=\int_{E}\left(\int_{E} G(y, x) \eta(d y)\right) v(d x)=\int_{E} G_{\beta} v(x) \eta(d x) .
$$


For each $t>0$, let $\eta_{t}$ be the $\beta$-co-equilibrium measure of the quasi-open set $\{x$ : $|u(x)|>t\}$. Note that $\widehat{G}_{\beta} \eta_{t} \geq 1$ on $\{x:|u(x)|>t\}$ and $\eta_{t}(E)=\widehat{\operatorname{Cap}}_{(\beta)}(\{|u(x)|>$ $t\})$. By (4.3) and Proposition 4.1.

$$
\begin{aligned}
\int_{E} u(x)^{2} v(d x) & =2 \int_{0}^{\infty}\left(t \int_{E} 1_{\{x:|u(x)|>t\}} v(d x)\right) d t \\
& \leq 2 \int_{0}^{\infty}\left(t \int_{E} \widehat{G}_{\beta} \eta_{t}(x) v(d x)\right) d t \\
& =2 \int_{0}^{\infty}\left(t \int_{E} G_{\beta} v(x) \eta_{t}(d x)\right) d t \\
& \leq 2\left\|G_{\beta} v\right\|_{\infty} \int_{0}^{2} 2 t \widehat{\operatorname{Cap}}_{(\beta)}(\{|u(x)|>t\}) \\
& \leq 4 K\left\|G_{\beta} v\right\|_{\infty} \mathcal{E}_{\beta}(u, u) .
\end{aligned}
$$

Remark 5. (1) In [18], Fitzsimmons proved the inequality in this proposition with a different constant in the semi-Dirichlet context. His proof is different from ours. (2) When $X$ is symmetric, the sector constant $K$ in 4.1 is 1 and in this case the above Proposition was proved in [44]. We note that the coefficient $4 K$ in Proposition 4.2 is not optimal. In [42], Stollman and Voigt showed that if $X$ is symmetric then

$$
\int_{E} u^{2}(x) v(d x) \leq\left\|G_{\beta} v\right\|_{\infty} \mathcal{E}_{\beta}(u, u) \quad \text { for } u \in \mathcal{F} .
$$

For non-symmetric processes $X$, if we use Rao's capacity estimate, that is, if we use Proposition 4.1 with 4 in place of $2 K$ (see Remark 4), then we have

$$
\int_{E} u^{2}(x) v(d x) \leq 8\left\|G_{\beta} v\right\|_{\infty} \mathcal{E}_{\beta}(u, u) \quad \text { for } u \in \mathcal{F} .
$$

(3) Using an argument similar to the proofs of Propositions 2.2 and 3.1 in [4], one can show that

$$
\int_{E} u^{2}(x) v(d x) \leq\left\|G_{\beta} v\right\|_{\infty}^{1 / 2}\left\|\widehat{G}_{\beta} v\right\|_{\infty}^{1 / 2} \mathcal{E}_{\beta}(u, u)
$$

for $u \in \mathcal{F}$.

Lemma 4.3. (1) If $v \in \mathbf{K}(X)$, then for any $\varepsilon>0$, there is a constant $A_{\varepsilon}>0$ such that

$$
\int_{E} f^{2}(x)|\nu|(d x) \leq \varepsilon \mathcal{E}(f, f)+A_{\varepsilon} \int_{E} f^{2}(x) m(d x), \quad f \in \mathcal{F} .
$$

(2) If $v \in \mathbf{K}_{1}(X)$ with $\beta(v)<\max \left\{\frac{1}{4 K}, \frac{1}{8}\right\}$, then there are constants $\lambda \in(0,1)$ and $A>0$ such that

$$
\int_{E} f^{2}(x)|v|(d x) \leq \lambda \mathcal{E}(f, f)+A \int_{E} f^{2}(x) m(d x), \quad f \in \mathcal{F} .
$$

Here $K$ is the constant in (4.1) and $\beta(v)$ is the constant in the definition of $\mathbf{K}_{1}(X)$. 
Proof. (1) follows immediately from Proposition 4.2 and the fact $v \in \mathbf{K}(X)$ is equivalent to $\lim _{\beta \uparrow \infty} \sup _{x} G_{\beta}|\nu|(x)=0$.

(2) Without loss of generality, we assume that $v \in \mathbf{K}_{1}(X)$ with $\beta(v)<$ $\max \left\{\frac{1}{4 K}, \frac{1}{8}\right\}$ is a positive measure. Let $A_{t}^{v}$ be the continuous additive functional of $X$ whose Revuz measure is $v$. It can be shown similar to that of Proposition 2.2 in [13] that there is a $t_{0}>0$ such that

$$
\theta:=\sup _{x \in E} \mathbf{E}_{x}\left[A_{t_{0}}^{v}\right]<\max \left\{\frac{1}{4 K}, \frac{1}{8}\right\} .
$$

This implies that

$$
\lim _{\beta \rightarrow \infty}\left\|G_{\beta} \nu\right\|_{\infty}=\lim _{\beta \rightarrow \infty} \sup _{x \in E} \mathbf{E}_{x}\left[\int_{0}^{\infty} e^{-\beta t} d A_{t}^{\nu}\right] \leq \theta<\max \left\{\frac{1}{4 K}, \frac{1}{8}\right\} .
$$

Lemma 4.3(2) now follows from Proposition 4.2 and (4.4) in Remark 5.

When the process $X$ is symmetric, Lemma 4.3(1) was proved in [1].

The following result follows immediately from Lemma 4.3, the definition of $\mathbf{J}(X)$, and the inequality $|f(x) f(y)| \leq\left(f^{2}(x)+f^{2}(y)\right) / 2$.

Lemma 4.4. If $F \in \mathbf{J}(X)$ is such that $\widehat{F}(x, y)=F(y, x)$ is in $\mathbf{J}(\widehat{X})$, then for any $\varepsilon>0$, there is a constant $A_{\varepsilon}>0$ such that

$$
\begin{aligned}
& \int_{E} \int_{E}|f(x) f(y)|\left|e^{F(y, x)}-1\right| N(x, d y) \mu_{H}(d x) \leq \varepsilon \mathcal{E}(f, f) \\
& \quad+A_{\varepsilon} \int_{E} f^{2}(x) m(d x), \quad f \in \mathcal{F} .
\end{aligned}
$$

In the remainder of this section, we fix a function $F \in \mathbf{J}(X)$ with $\widehat{F} \in \mathbf{J}(\widehat{X})$ and a signed measure $v$ such that $|v|=v^{+}+v^{-}$is in $\mathbf{K}(X) . F$ can and will be extended to $E_{\partial} \times E_{\partial}$ by setting it to be zero off $E \times E$. Then it follows from the above two lemmas that the bilinear form $(\mathcal{Q}, \mathcal{F})$ defined by

$$
\begin{aligned}
\mathcal{Q}(f, g)= & \mathcal{E}(f, g)-\int_{E} f(x) g(x) v(d x) \\
& -\int_{E} \int_{E} f(x) g(y)\left(e^{F(y, x)}-1\right) N(x, d y) \mu_{H}(d x)
\end{aligned}
$$

is closed, lower bounded and satisfies sector condition, i.e., there are positive constants $c$ and $\beta$ such that $\mathcal{Q}_{\beta}(f, f) \geq 0$ for $f \in \mathcal{F}$ and

$$
\left|\mathcal{Q}_{\beta}(f, g)\right| \leq c \mathcal{Q}_{\beta}(f, f)^{1 / 2} \mathcal{Q}_{\beta}(g, g)^{1 / 2} \quad f, g \in \mathcal{F} .
$$

Let $A_{t}=A_{t}^{+}-A_{t}^{-}$, where $A_{t}^{+}$and $A_{t}^{-}$are the positive continuous additive functionals of $X$ associated with $v^{+}$and $v^{-}$respectively. Put $e_{v+F}(t)=\exp \left(A_{t}+\right.$ $\left.A_{F}(t)\right)$. For any nonnegative $f$ and $t \geq 0$, we define the operators $T_{t}$ and $\widehat{T}_{t}$ by

$$
T_{t} f(x)=\mathbf{E}_{x}\left[e_{v+F}(t) f\left(X_{t}\right)\right], \quad x \in E,
$$


and

$$
\widehat{T}_{t} f(x)=\widehat{\mathbf{E}}_{x}\left[e_{v+\widehat{F}}(t) f\left(\widehat{X}_{t}\right)\right], \quad x \in E .
$$

It follows from Proposition 2.3, there exist positive constants $c_{1}, c_{2}$ and $\beta_{1}, \beta_{2}$ such that for all $t \geq 0$,

$$
\begin{gathered}
\sup _{x \in E} \mathbf{E}_{x}\left[e_{|v|+|F|}(t)\right] \leq c_{1} e^{\beta_{1} t} . \\
\sup _{x \in E} \mathbf{E}_{x}\left[e_{2|v|+2|F|}(t)\right] \leq c_{2} e^{\beta_{2} t} .
\end{gathered}
$$

Note that $T_{t}$ and $\widehat{T}_{t}$ are bounded operators in $L^{2}(E, m)$. To see this, note that for $f \in L^{2}(E, m)$,

$$
\left|T_{t} f(x)\right|^{2} \leq \mathbf{E}_{x}\left[e_{2|v|+2|F|}(t)\right] \mathbf{E}_{x}\left[f\left(X_{t}\right)^{2}\right] .
$$

Hence

$$
\left\|T_{t} f\right\|_{2} \leq c_{2} e^{\beta_{2} t}\|f\|_{2} .
$$

In fact one can easily show, similar to that in [15], that for any $1 \leq p \leq \infty,\left(T_{t}\right)$ and $\left(\widehat{T}_{t}\right)$ are semigroups on $L^{p}(E, m)$ and that, for any $1 \leq p<\infty,\left(T_{t}\right)$ and $\left(\widehat{T}_{t}\right)$ are strongly continuous semigroups on $L^{p}(E, m)$. Furthermore, $\left(\widehat{T}_{t}\right)$ is the dual semigroup of $\left(T_{t}\right)$ on $L^{2}(E, m)$. To prove this fact, we recall the definition of the time-reversal operator $r_{t}$ on the path space. Given a path $\omega \in\{t<\zeta\}$, the operator $r_{t}$ is defined by

$$
r_{t}(\omega)(s)= \begin{cases}\omega\left((t-s)_{-},\right. & \text {if } 0 \leq s \leq t, \\ \omega(0), & \text { if } s \geq t .\end{cases}
$$

Here for $r>0, \omega(r)_{-}:=\lim _{s \uparrow r} \omega(s)$.

Theorem 4.5. For any $f, g \in L^{2}(E, m)$ and $t>0$, we have

$$
\int_{E} g(x) T_{t} f(x) m(d x)=\int_{E} f(x) \widehat{T}_{t} g(x) m(d x) .
$$

Proof. By time reversal, we have

$$
\begin{aligned}
\int_{R^{d}} g(x) T_{t} f(x) d x & =\int_{R^{d}} g(x) \mathbf{E}_{x}\left[e_{\nu, F}(t) f\left(X_{t}\right)\right] d x \\
& =\mathbf{E}_{m}\left[g\left(X_{0}\right) e_{\nu+F}(t) f\left(X_{t}\right)\right] \\
& =\widehat{\mathbf{E}}_{m}\left[f\left(\widehat{X}_{0}\right) e_{\nu+F}(t) \circ r_{t} g\left(\widehat{X}_{t}\right)\right] .
\end{aligned}
$$

From the definition of $e_{\nu+F}(t)$, it was easy to see that $e_{\nu+F}(t) \circ r_{t}=e_{\nu+\widehat{F}}(t)$, hence we have shown that

$$
\int_{E} g(x) T_{t} f(x) m(d x)=\int_{E} f(x) \widehat{T}_{t} g(x) m(d x) .
$$

Put $\beta_{0}=\beta_{1} \vee \beta_{2}$, where $\beta_{1}$ and $\beta_{2}$ are the constants in (4.6)-(4.7). Then for any $\beta>\beta_{0}$, the map

$$
f \mapsto \mathbf{E} .\left[\int_{0}^{\infty} e^{-\beta t} e_{\nu+F}(t) f\left(X_{t}\right) d t\right]
$$

maps $L^{\infty}(E, m)$ into $L^{\infty}(E, m)$ and maps $L^{2}(E, m)$ into $L^{2}(E, m)$. 
Theorem 4.6. If $f \in L^{2}(E, m) \cap L^{\infty}(E, m)$, then for any $\beta>\beta_{0}$, the function

$$
u(x)=\mathbf{E}_{x}\left[\int_{0}^{\infty} e^{-\beta t} e_{\nu+F}(t) f\left(X_{t}\right) d t\right]
$$

is a quasi-continuous function in $\mathcal{F}$ such that for any quasi-continuous $v \in \mathcal{F}$,

$$
\begin{aligned}
\mathcal{E}_{\beta}(u, v)= & \int_{E} f(x) v(x) m(d x)+\int_{E} u(x) v(x) v(d x) \\
& +\int_{E} \int_{E} v(x)\left(e^{F(x, y)}-1\right) u(y) N(x, d y) \mu_{H}(d x) .
\end{aligned}
$$

Proof. Put

$$
\begin{aligned}
\eta(d x)= & |\nu|(d x)+\int_{E}\left(e^{|F(x, y)|}-1\right) N(x, d y) \mu_{H}(d x) \\
& +\int_{E}\left(e^{|\hat{F}(x, y)|}-1\right) \widehat{N}(x, d y) \mu_{H}(d x) .
\end{aligned}
$$

Let $S_{0}$ denote the space of positive Radon measures $\mu$ on $E$ of finite energy integral (that is, there is a constant $c>0$ such that $\int_{E}|v(x)| \mu(d x) \leq c \mathcal{E}_{1}(v, v)^{1 / 2}$ for every $v \in \mathcal{F}$ ). It is well known (cf. Lemma 4.1 .7 of [35]) that there is a sequence of compact sets $K_{n}$ such that (i) $1_{K_{n}}(x) \eta(d x)$ is in $S_{0}$ for each $n$, (ii) $\eta\left(E \backslash \cup_{n=1}^{\infty} K_{n}\right)=0$, (iii) $\lim _{n \rightarrow \infty} \operatorname{Cap}_{(1)}\left(K \backslash K_{n}\right)=0$ for any compact set $K$. For any $n$, put $v_{n}(d x)=1_{K_{n}}(x) v(d x)$ and $F_{n}(x, y)=1_{K_{n} \times K_{n}}(x, y) F(x, y)$. Define

$$
u_{n}(x)=\mathbf{E}_{x}\left[\int_{0}^{\infty} e^{-\beta t} e_{v_{n}+F_{n}}(t) f\left(X_{t}\right) d t\right] \text {. }
$$

Note that by (4.6), $u_{n}$ and $u$ are uniformly bounded. Using the argument leading to (4.8), one can easily show that $u_{n}$ converges to $u$ pointwise and in $L^{2}$ on $E$. From the proof of Theorem 2.4 we see that

$$
u_{n}(x)=G_{\beta} f(x)+G_{\beta}\left(\left(\mu_{H} \mathbf{F}_{\mathbf{n}}+v_{n}\right) u_{n}\right)(x) .
$$

Therefore, for each $n, u_{n}$ is a quasi-continuous function in $\mathcal{F}$ such that for any quasi continuous $v \in \mathcal{F}$,

$$
\begin{aligned}
\mathcal{E}\left(u_{n}, v\right) & +\beta \int_{E} u_{n}(x) v(x) m(d x) \\
= & \int_{E} f(x) v(x) m(d x)+\int_{E} u_{n}(x) v(x) v_{n}(d x) \\
& +\int_{E} \int_{E} v(x)\left(e^{F_{n}(x, y)}-1\right) u_{n}(y) N(x, d y) \mu_{H}(d x) .
\end{aligned}
$$

It follows from this identity and Lemmas 4.3 and 4.4 that

$$
\mathcal{E}\left(u_{n}, u_{n}\right) \leq\|f\|_{2}\left\|u_{n}\right\|_{2}+\frac{1}{2} \mathcal{E}\left(u_{n}, u_{n}\right)+C\left\|u_{n}\right\|_{2}^{2}
$$

where $C$ is a positive constant. Therefore we have

$$
\sup _{n} \mathcal{E}_{1}\left(u_{n}, u_{n}\right)<\infty \text {. }
$$


By (4.9), Lemmas 4.3 and 4.4 and Proposition 4.2,

$$
\begin{aligned}
& \mathcal{E}_{\beta}\left(u_{n+l}-u_{n}, u_{n+l}-u_{n}\right) \\
&= \int_{E}\left(u_{n+l}-u_{n}\right)(x)\left(\left(\mathbf{F}_{n+l} u_{n+l}(x)-\mathbf{F}_{n} u_{n}(x)\right) \mu_{H}(d x)\right. \\
&\left.+u_{n+l} v_{n+l}(d x)-u_{n}(x) v_{n}(d x)\right) \\
&= \int_{K_{n} \times K_{n}}\left(u_{n+l}-u_{n}\right)(x)\left(u_{n+l}-u_{n}\right)(y)\left(e^{F(x, y)}-1\right) N(x, d y) \mu_{H}(d x) \\
& \int_{\left(K_{n+l} \times K_{n+l}\right) \backslash\left(K_{n} \times K_{n}\right)}\left(u_{n+l}-u_{n}\right)(x) u_{n+l}(y)\left(e^{F(x, y)}-1\right) N(x, d y) \mu_{H}(d y) \\
& \quad+\int_{K_{n}}\left(u_{n+l}(x)-u_{n}(x)\right)^{2} v(d x)+\int_{K_{n+l} \backslash K_{n}}\left(u_{n+l}-u_{n}\right)(x) u_{n+l}(x) v(d x) \\
& \leq \frac{1}{2} \mathcal{E}_{\beta}\left(u_{n+l}-u_{n}, u_{n+l}-u_{n}\right)+C\left\|u_{n+l}-u_{n}\right\|_{2}^{2} \\
&+2 K\left\|G_{\beta}\left(1_{K_{n+l} \backslash K_{n}} \eta\right)\right\|_{\infty}\left(\mathcal{E}_{\beta}\left(u_{n+l}, u_{n+l}\right)+\mathcal{E}_{\beta}\left(u_{n+l}-u_{n}, u_{n+l}-u_{n}\right)\right),
\end{aligned}
$$

where $C$ is a positive constant. Hence

$$
\begin{aligned}
& \mathcal{E}_{\beta}\left(u_{n+l}-u_{n}, u_{n+l}-u_{n}\right) \\
& \quad \leq 2 C\left\|u_{n+l}-u_{n}\right\|_{2}^{2}+12 K\left\|G_{\beta}\left(1_{K_{n+l} \backslash K_{n}} \eta\right)\right\|_{\infty} \sup _{n \geq 1} \mathcal{E}_{\beta}\left(u_{n}, u_{n}\right),
\end{aligned}
$$

which converges to zero as $n \rightarrow \infty$. This is because $u_{n}$ converges to $u$ in $L^{2}(E, m)$ and by Proposition 2.1 $\left\|G_{\beta}\left(1_{K_{n+l} \backslash K_{n}} v\right)\right\|_{\infty} \rightarrow 0$ as $n \rightarrow \infty$. Thus $\left\{u_{n}\right\}_{n \geq 1}$ is an $\mathcal{E}_{\beta}$-Cauchy sequence in $\mathcal{F}$, which must converge to $u$. Letting $n \rightarrow \infty$ in (4.10) proves the theorem.

Theorem 4.7. For $\beta>\beta_{0}$ and $f \in L^{2}(E, m)$,

$$
u(x)=\mathbf{E}_{x}\left[\int_{0}^{\infty} e^{-\beta t} e_{\nu+F}(t) f\left(X_{t}\right) d t\right]
$$

is a quasi continuous function in $\mathcal{F}$ such that for any $v \in \mathcal{F}$,

$$
\begin{aligned}
\mathcal{E}_{\beta}(u, v)= & \int_{E} f(x) v(x) m(d x)+\int_{E} u(x) v(x) v(d x) \\
& +\int_{E} \int_{E} v(x)\left(e^{F(x, y)}-1\right) u(y) N(x, d y) \mu_{H}(d x) .
\end{aligned}
$$

Proof. For $n \geq 1$, let $f_{n}(x)=f(x) 1_{\{|f(x)| \leq n\}}(x)$ and define

$$
u_{n}(x)=\mathbf{E}_{x}\left[\int_{0}^{\infty} e^{-\beta t} e_{\nu+F}(t) f_{n}\left(X_{t}\right) d t\right] .
$$

By the previous theorem, $u_{n}$ is a quasi-continuous function in $\mathcal{F}$ such that for any $v \in \mathcal{F}$,

$$
\begin{aligned}
\mathcal{E}_{\beta}\left(u_{n}, v\right)= & \int_{E} f(x) v(x) m(d x)+\int_{E} u_{n}(x) v(x) v(d x) \\
& +\int_{E} \int_{E} v(x)\left(e^{F(x, y)}-1\right) u_{n}(y) N(x, d y) \mu_{H}(d x) .
\end{aligned}
$$


From (4.8), we know that $u_{n}$ converges to $u$ in $L^{2}(E, m)$. It follows from the identity above, Lemmas 4.3 and 4.4 that

$\mathcal{E}\left(u_{n}-u_{m}, u_{n}-u_{m}\right) \leq\|f\|_{2}\left\|u_{n}-u_{m}\right\|_{2}+\frac{1}{2} \mathcal{E}\left(u_{n}-u_{m}, u_{n}-u_{m}\right)+C\left\|u_{n}-u_{m}\right\|_{2}^{2}$

where $C$ is a positive constant. Therefore $u_{n}$ is a Cauchy sequence with respect to $\mathcal{E}$. Since $u_{n}$ converges to $u$ in $L^{2}(E, m)$, we have that $u_{n}$ converges to $u$ with respect to $\mathcal{E}_{1}$. The conclusions of the theorem now follows immediately.

The above theorem tells us

Theorem 4.8. $\left\{T_{t}, t \geq 0\right\}$ is the unique strongly continuous semigroup on $L^{2}(E, m)$ associated with the bilinear form $(\mathcal{Q}, \mathcal{F})$.

The following result follows immediately from the above theorem (cf. [33]).

Corollary 4.9. The $L^{2}$-infinitesimal generator $\left(\mathcal{A}, D(\mathcal{A})\right.$ of $\left\{T_{t}, t \geq 0\right\}$ is

$$
\begin{gathered}
D(\mathcal{A})=\left\{u \in \mathcal{F}: \text { there is some } f \in L^{2}(E, m) \text { such that } \mathcal{Q}(u, v)\right. \\
\left.=-\int_{E} f(x) v(x) m(d x) \text { for each } v \in \mathcal{F}\right\}, \\
\mathcal{A} u=f \quad \text { for } u \in D(\mathcal{A}) \text { with above } f .
\end{gathered}
$$

Remark 6. In fact, Theorems 4.6 and 4.8 holds for $v \in \mathbf{K}_{1}(X)$ with $\beta(v)<$ $\max \left\{\frac{1}{4 K}, \frac{1}{8}\right\}$ and $F \in \mathbf{J}(X)$ as well. This is because by Lemma 4.3(2), the bilinear form $(\mathcal{Q}, \mathcal{F})$ defined by $(4.5)$ is closed, lower bounded and satisfies the sector condition. It is can be shown (see Theorem 3.14 of Getoor [24]) that the Schrödinger semigroup $T_{t}$ defined by $T_{t} f(x)=\mathbf{E}_{x}\left[e_{v+F}(t) f\left(X_{t}\right)\right]$ is a strongly continuous semigroup in $L^{2}(E, m)$. Theorems $4.6,4.7$ and therefore 4.8 can then be proved with an easy modification of the corresponding proofs in this section.

Acknowledgements. Part of the results in this paper has been presented at the Laboratoire de Statistique et Probabilites at l'Universite Paul-Sabatier, at the conference "New trends in Potential Theory and Applications" held at the ZiF, Universität Bielefeld, and at the Workshop on Lévy Processes held at University of Warwick. The first named author thanks Michel Ledoux, Zhongmin Qian, and the organizers of the two conferences for the invitation and the warm hospitality. He also thanks Wolfhard Hansen for telling him the $G$-Kato measures introduced in his joint paper [4] with Amor and for remarks on its possible equivalence with our $\mathbf{K}_{\infty}(X)$ class. This resulted in Proposition 2.1. We are grateful to Pat Fitzsimmons for his very helpful comments about this paper. Thanks are also extended to the referee for a very careful reading of the paper and very helpful comments.

\section{References}

[1] Albeverio, S., Ma, Z.: Perturbation of Dirichlet forms - Lower semiboundedness, closability and form cores, J. Funct. Ana., 99, 332-356 (1991)

[2] Albeverio, S., Ma, Z.: Additive functionals, nowhere Radon and Kato class smooth measures associated with Dirichlet forms, Osaka J. Math., 29, 247-265 (1992)

[3] Albeverio, S., Ma, Z., Röckner, M.: Quasi-regular Dirichlet forms and Markov processes. J. Funct. Anal., 111, 118-154 (1993) 
[4] Amor, A.B., Hansen, W.: Continuity of eigenvalues for Schrödinger operators, $L^{p}$ properties of Kato type integral operators. Math. Ann. 321, 925-953 (2001)

[5] Benveniste, A., Jacod, J.: Systèmes de Lévy des processus de Markov. Invent. Math., 21, 183-198 (1973)

[6] Blumenthal, R.M., Getoor, R.K.: Markov Processes and Potential Theory. Academic Press: New York, 1968

[7] Bogdan, K., Byczkowski, T.: Potential theory for the $\alpha$-stable Schrödinger operator on bounded Lipschitz domains. Studia Math., 133, 53-92 (1999)

[8] Chen, Z.-Q.: Gaugeability and conditional gaugeability, Trans. Amer. Math. Soc. (to appear), 2001

[9] Chen, Z.-Q., Ma, Z.-M., Röckner, M.: Quasi-homeomorphisms of Dirichlet forms. Nagoya Math. J., 136, 1-15 (1994)

[10] Chen, Z.-Q., Song, R.: Estimates on Green functions and Poisson kernels of symmetric stable processes. Math. Ann., 312, 465-601 (1998)

[11] Chen, Z.-Q., Song, R.: Intrinsic ultracontractivity and conditional gauge for symmetric stable processes. J. Funct. Anal., 150, 204-239 (1997)

[12] Chen, Z.-Q., Song, R.: Intrinsic ultracontractivity, conditional lifetimes and conditional gauge for symmetric stable processes on rough domains. Ill. J. Math., 44, 138-160 (2000)

[13] Chen, Z.-Q., Song, R.: General gauge and conditional gauge theorems. Ann. Probab. (to appear), 2001

[14] Chung, K.L., Rao, M.: General gauge theorem for multiplicative functionals. Trans. Amer. Math. Soc. 306, 819-836 (1988)

[15] Chung, K.L., Zhao, Z.: From Brownian motion to Schrödinger's equation. Springer: Berlin, 1995

[16] Fitzsimmons, P.J.: On the excursions of Markov processes in classical duality. Probab. Theory Related Fields, 75, 159-178 (1987)

[17] Fitzsimmons, P.J.: Markov processes and nonsymmetric Dirichlet forms without regularity. J. Funct. Anal., 85, 287-306 (1989)

[18] Fitzsimmons, P.J.: On the quasi-regularity of semi-Dirichlet forms. Potential Analysis, 15, 151-185 (2001)

[19] Fitzsimmons, P.J., Getoor, R.K.: Revuz measures and time changes. Math. Z., 199, 233-256 (1988)

[20] Fitzsimmons, P.J., Getoor, R.K.: Smooth measures and continuous additive functionals of right Markov processes. In "Ito's Stochastic Calculus and Probability Theory", N. Ikeda, S. Watanabe, M. Fukushima and H. Kunita (eds.) Springer-Verlag Tokyo, 1996

[21] Fukushima, M., Oshima, Y., Takeda, M.: Dirichlet forms and symmetric Markov processes. Walter de Gruyter, Berlin, 1994

[22] Getoor, R.K.: Duality of Lévy systems. Z. Wahrs. verw. Geb., 19, 257-270 (1971)

[23] Getoor, R.K.: Transience and recurrence of Markov processes. In Séminaire de Probabilités XIV, Lect. Notes Math., 784, 397-409 (1980)

[24] Getoor, R.K.: Measure perturbations of Markov semigroups. Potential Analysis, 11, 101-133 (1999)

[25] Getoor, R.K., Glover, J.: Riesz decompositions in Markov process theory. Trans. Amer. Math. Soc., 285, 107-132 (1984)

[26] Hansson, K.: Imbedding theorems of Sobolev type in potential theory. Math. Scand., 45, 77-102 (1979)

[27] He, S.W., Wang, J.G., Yan, J.A.: Semimartingale Theory and Stochastic Calculus. Science Press, Beijing, New York, 1992

[28] Janicki, A., Weron, A.: Simulation and Chaotic Behavior of $\alpha$-Stable Processes. Dekker, 1994 
[29] Kim, J.H.: Stochastic calculus related to non-symmetric Dirichlet forms, Osaka J. Math, 24, 331-371 (1987)

[30] Kolsrud, T.: Capacitary integrals in Dirichlet spaces. Math. Scand. 55, 95-120 (1984)

[31] Kulczycki, T.: Properties of Green function of symmetric stable processes. Probab. Math. Stat., 17(2), 339-364 (1997)

[32] Kunita, H., Watanabe, T.: Notes on transformations of Markov processes connected with multiplicative functionals. Mem. Fac. Sci. Kyushu Univ. Ser. A, 17, 181-191 (1963)

[33] Ma, Z.-M., Röckner, M.: Introduction to the Theory of (Non-symmetric) Dirichlet Forms. Springer-Verlag: Berlin, 1992

[34] Maz'ya, V.G.: Sobolev Spaces. Springer-Verlag: Berlin, 1985

[35] Oshima, Y.: Lectures on Dirichlet Spaces, (unpublished) Lectures Notes given in Erlangen, 1988

[36] Rao, M.: Capacitary inequality for energy, Israel J. Math., 61, 179-191 (1988)

[37] Samorodnitsky, G., Taqqu, M.S.: Stable Non-Gaussian Random Processes. Chapman \& Hall, New York-London, 1994

[38] Sharpe, M.: General Theory of Markov Processes, Academic Press, Boston, 1988

[39] Song, R.: Probabilistic approach to the Dirichlet problem of perturbed stable processes, Prob. Th. Rel Fields, 95, 371-389 (1993)

[40] Song, R.: Feynman-Kac semigroup with discontinuous additive functionals, J. Theoretical probab., 8, 727-762 (1995)

[41] Song, R., Wu, J.-M.: Boundary Harnack Principle for symmetric stable processes. J. Funct. Anal., 168, 403-427 (1999)

[42] Stollman, P., Voigt, J.: Perturbation of Dirichlet forms by measures, Potential Analysis, 5, 109-138 (1996)

[43] Sturm, K.-T.: Gauge theorems for resolvents with application to Markov process. Probab. Th. Rel. Fields 89, 387-406 (1991)

[44] Vondracek, Z.: An estimate for the $L^{2}$-norm of a quasi continuous function with respect to a smooth measure, Arch. Math., 67, 408-414 (1996)

[45] Ying, J.: Bivariate Revuz measures and the Feynman-Kac formula. Ann. Inst. Henri Poincaré, 32, 251-287 (1998)

[46] Zhao, Z.: Green function for Schrödinger operator and conditioned Feynman-Kac gauge. J. Math. Anal. Appl., 116, 309-334 (1986) 